\title{
Mitochondria as Target for Tumor Management of Hemangioendothelioma
}

\author{
Gayle M. Gordillo,, ${ }^{1, *}$ Ayan Biswas, ${ }^{1, *}$ Kanhaiya Singh,, Abhishek Sen, ${ }^{1}$ Poornachander R. Guda, \\ Caroline Miller, ${ }^{2}$ Xueliang Pan, ${ }^{3}$ Savita Khanna, ${ }^{1}$ Enrique Cadenas, ${ }^{4}$ and Chandan K. Sen ${ }^{1,5}$
}

\begin{abstract}
Aims: Hemangioendothelioma (HE) may be benign or malignant. Mouse hemangioendothelioma endothelial (EOMA) cells are validated to study mechanisms in HE. This work demonstrates that EOMA cells heavily rely on mitochondria to thrive. Thus, a combination therapy, including weak X-ray therapy (XRT, 0.5 Gy) and a standardized natural berry extract (NBE) was tested. This NBE is known to be effective in managing experimental HE and has been awarded with the Food and Drug Administration Investigational New Drug (FDAIND) number 140318 for clinical studies on infantile hemangioma.

Results: NBE treatment alone selectively attenuated basal oxygen consumption rate of EOMA cells. NBE specifically sensitized EOMA, but not murine aortic endothelial cells to XRT-dependent attenuation of mitochondrial respiration and adenosine triphosphate (ATP) production. Combination treatment, selectively and potently, influenced mitochondrial dynamics in EOMA cells such that fission was augmented. This was achieved by lowering of mitochondrial sirtuin 3 (SIRT3) causing increased phosphorylation of AMP-activated protein kinase (AMPK). A key role of SIRT3 in loss of EOMA cell viability caused by the combination therapy was evident when pyrroloquinoline quinone, an inducer of SIRT3, pretreatment rescued these cells.

Innovation and Conclusion: Mitochondria-targeting NBE significantly extended survival of HE-affected mice. The beneficial effect of NBE in combination with weak X-ray therapy was, however, far more potent with threefold increase in murine survival. The observation that safe natural products may target tumor cell mitochondria and sharply lower radiation dosage required for tumor management warrants clinical testing. Antioxid. Redox Signal. 34, 137-153.
\end{abstract}

Keywords: hemangioendothelioma, mitochondria, radiation, tumor, therapy

Introduction

$\mathbf{C}$ LINICALly PRESENTED AS vascular neoplasms, hemangioendothelioma (HE) may also display characteristics intermediate between entirely benign hemangiomas and highly malignant angiosarcomas $(38,59,63)$. In children, they frequently cause deformity and can cause death. Kaposiform HE features several solid poorly circumscribed nodules such that each nodule is composed of a mixture of small capillaries and solid lobules of endothelial cells arranged in a glomeruloid pattern. Mouse hemangioendothelioma endothelial (EOMA) cells are a validated experimental model to study mechanisms in $\mathrm{HE}$ and its management $(11,12,23)$.

Mitochondrial functionality is a critical requirement for tumor anabolism. The growth of neoplastic tissue is supported by a hyperactive glycolytic machinery, often anaerobic. It is commonly accepted that pyruvate, thus generated in the cytoplasm, is shunted to produce lactate via lactate dehydrogenase (LDH). It has been thus assumed that tumor

\footnotetext{
${ }^{1}$ Department of Surgery, Indiana Center for Regenerative Medicine and Engineering, Indiana University School of Medicine, Indianapolis, Indiana, USA.

${ }^{2}$ Electron Microscopy Core, Indiana University School of Medicine, Indianapolis, Indiana, USA.

${ }^{3}$ Department of Biomedical Informatics, The Ohio State University, Columbus, Ohio, USA.

${ }^{4}$ Department of Pharmacology and Pharmaceutical Sciences, School of Pharmacy, University of Southern California, Los Angeles, California, USA.

${ }^{5}$ Weldon School of Biomedical Engineering, Purdue University, West Lafayette, Indiana, USA.

*Both these authors contributed equally to this work.
} 


\section{Innovation}

This work shows that the mitochondrial dynamics of tumor cells, not non-tumor cells, can be specifically targeted by a standardized (FDA-IND No. 140318) natural product. Interestingly, this work provides first evidence that such natural products may be utilized in combination to lower the radiation dose for tumor therapy.

cells are limited in their capacity for oxidative phosphorylation (OXPHOS). Such notion would assign low value to mitochondrial functionality for its significance in supporting tumor anabolism (51). More recent work, however, appreciates that tumor metabolism is less homogenous than was previously imagined (51). Thorough characterization of the metabolic process involved in tumor has now identified tumorigenic cell populations that are heavily reliant on mitochondrial respiration compared with their dependence on glycolysis. Mitochondrial depletion of cancer cells markedly limited tumorigenic potential $(13,25,37,60)$. Loss of mitochondrial respiration causes proliferating cancer cells to become functionally limited for electron acceptors (50).

Recent literature suggests an essential role of mitochondria in cancer. Cancer cells devoid of mitochondrial DNA (mtDNA) lose tumorigenic potential unless OXPHOS is reconstituted in these cells by mitochondria acquired from host stroma (7). Furthermore, defects in OXPHOS sensitized tumor cells to cytotoxic drugs $(13,53)$. Conventional radiation therapy is known to compromise tumor mitochondrial function (44). Targeting mitochondrial function has emerged as a viable strategy for the development of novel anticancer agents. Cytotoxic drugs, the cornerstone for chemotherapy, however, are notorious for their adverse side effects. Furthermore, many of these cytotoxic drugs are classified by the National Institute for Occupational Safety and Health (NIOSH) as hazardous drugs as they threaten the health of an estimated 8 million U.S. health care workers.

Berry polyphenols have been recognized for their chemopreventive properties $(40,52)$. Low fruit and vegetable intake is a known contributor to cancer-related deaths $(5,58)$. On the contrary, specific phytochemicals such as anthocyanins are known to act on a number of specific molecular mechanisms that inhibit tumorigenesis (56). Our recent work has identified that a standardized anthocyanin-rich natural berry extract (NBE) is effective in prolonging survival of $\mathrm{HE}$ bearing mice (11). The objective of this work is to investigate tumor mitochondrial function and its significance in the development of $\mathrm{HE}$, as well as in the survival of HE-bearing mice. NBE and therapeutic gamma X-ray radiation (X-ray therapy $[\mathrm{XRT}]$ ) have been tested, alone or together in low dosages to study potential interaction, for their ability to target mitochondrial respiration in tumor cells.

\section{Results}

Sensitization of tumor forming endothelial cells to effects of XRT is time and dose dependent

To determine the dose and duration of NBE and XRT exposure, cell toxicity studies were performed. The NBE dose $(200 \mu \mathrm{g} / \mathrm{mL})$ and pretreatment duration were deter- mined using previously reported toxicity and dose/response studies for EOMA cells (4). Nontumor forming murine aortic endothelial (MAE) cells were used as controls $(11,12)$. Time and dose responses to XRT were analyzed using LDH and propidium iodide (PI) exclusion assays to identify the threshold dose of XRT needed to induce cell toxicity (Fig. 1a, b). XRT dose with 1.0 Gy (24 h) was toxic in MAE cells (Fig. 1a). This toxicity was, however, not observed using LDH leakage assay (Fig. 1b), which is known to be less sensitive than flow cytometry-based PI assay (15). EOMA cells, on the contrary, were sensitive to NBE treatment alone (Fig. 1a, b), as demonstrated by both PI exclusion and LDH leakage assays. Interestingly, NBE pretreatment sensitized EOMA cells to weak XRT (0.5 Gy)-mediated toxicity. Since weak XRT alone in EOMA cells did not induce toxicity, NBE pretreatment followed by a regimen of weak XRT was selected as the threshold condition to induce cell toxicity in EOMA cells as this treatment regimen was nontoxic for healthy MAE cells (Fig. 1a, b).

\section{NBE sensitized EOMA, not MAE, cells to weak XRT by inhibiting mitochondrial respiration}

Compared with MAE, EOMA cells displayed high basal mitochondrial oxygen consumption rate (OCR) (Fig. 2a). NBE treatment alone selectively attenuated basal OCR of EOMA cells. This reduction of OCR was not observed in MAE cells (Fig. 2a, d). Elevated basal OCR in EOMA, compared with MAE, was evident even after exposure to weak XRT. Interestingly, weak XRT was observed to offset the effect of NBE in attenuating mitochondrial respiration (Fig. 2b, d). Of note, this opposing effect of XRT on NBE was transient and did not hold over time (Fig. 2b, d). On the contrary, over time (6-12h), NBE significantly sensitized EOMA, but not MAE, cells to XRT (Fig. 2c, d). This observation was consistent with findings on EOMA cell toxicity in response to a combination of NBE and weak XRT (Fig. 1a, b). MAE cells remained safely unresponsive to such combination treatment (Fig. 2d).

\section{Inhibition of tumor cell glycolysis}

Extracellular acidification rate (ECAR) is a measure of cellular glycolytic rate (27). EOMA cells displayed threefold high basal ECAR than healthy MAE cells (Fig. 3a, d). Thus, glycolysis is hyperactive in EOMA cells. NBE successfully compromised the path of glycolytic energy supply specifically in EOMA, but not in MAE, cells at a lower dosage of radiation (Fig. 3a, d). This effect of NBE, taken together with its inhibitory effect of mitochondrial respiration as addressed above, does achieve substantial functional significance as reflected by the loss of EMOA cell viability (Fig. 1). As it relates to the study of healthy MAE, weak XRT accelerated glycolysis (Fig. 3b-d). In contrast, weak XRT had no effect on its own on EOMA cells (Fig. 3b-d). Interestingly, NBE was effective in inhibition of glycolysis specifically in EOMA cells. Of note, in combination with weak XRT, such inhibition of glycolysis was significantly potentiated (Fig. 3b-d).

\section{Blunted cellular respiration and reduced adenosine triphosphate production in EOMA cells}

Further studies tested the combination of NBE and weak XRT both of which are known to be safe for healthy MAE 

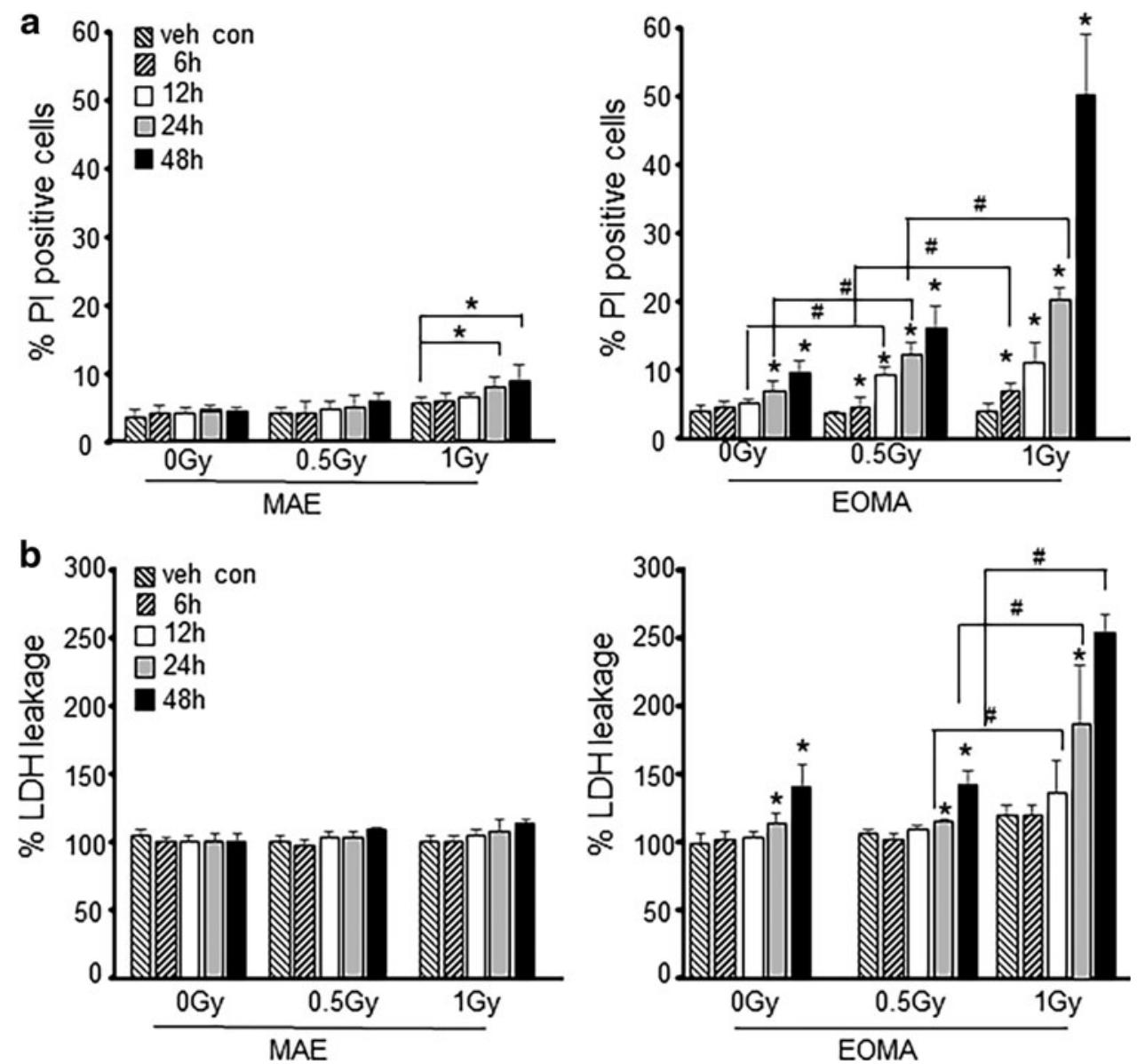

FIG. 1. Sensitization of tumor forming endothelial cells to effects of XRT is time and dose dependent. (a) EOMA and MAE cells were treated with NBE $(200 \mu \mathrm{g} / \mathrm{mL})$ and vehicle $(1 \%$ DMSO) for $6,12,24$, and $48 \mathrm{~h}$. In some treatment groups, 0.5 and 1 Gy of X-ray radiation was exposed for $6 \mathrm{~h}$ after NBE treatment schedule. Cell viability was measured by flow cytometry using PI (10 $\mu \mathrm{g} / \mathrm{mL})$ exclusion, and (b) LDH toxicity assay (MAK066; Sigma-Aldrich) shows a dose-dependent decrease in EOMA cell survival. MAE cells were included as nontumor forming endothelial cell controls. The key indicates the time of sample collection after XRT or after the 24-h pretreatment period for those samples that were not irradiated. ${ }^{*} p<0.05,{ }^{*} p<0.01, n=6$. DMSO, dimethyl sulfoxide; EOMA, mouse hemangioendothelioma endothelial; LDH, lactate dehydrogenase; MAE, murine aortic endothelial; NBE, natural berry extract; PI, propidium iodide; XRT, X-ray therapy.

(Fig. 1). The study of cellular bioenergetics showed that both NBE and XRT and their combination had no deleterious effect on MAE cells (Fig. 4a). Basal OCR in EOMA cells was fourfold higher than that in MAE. NBE inhibited OCR in EOMA but not in MAE cells (Fig. 4a). To dissect nonmitochondrial respiration from overall OCR, antimycin A and rotenone were used as inhibitors of complexes III and I, respectively (15). Residual oxygen consumption was twofold higher in EOMA compared with MAE cells (Fig. 4b). NBE treatment causes significant decrease in the residual oxygen consumption specifically in EOMA cells. Weak XRT alone was more effective than NBE in inhibiting nonmitochondrial respiration. However, a combination of NBE and weak XRT acted cooperatively to specifically inhibit nonmitochondrial respiration of EOMA cells (Fig. 4b).

Productivity of mitochondrial respiration in EOMA cells was determined by adenosine triphosphate (ATP) generated through OXPHOS in support of tumor anabolism. EOMA cells demonstrated a fivefold higher rate of ATP production than that in healthy MAE cells (Fig. 4c). Such high rate of ATP production remained unaffected by weak XRT. NBE was potent in blunting the rate of ATP production specifically in EOMA cells. How- ever, in combination with otherwise ineffective weak XRT, the effect of NBE was potentiated such that an estimated two-thirds of the rate of ATP production was inhibited (Fig. 4c). Although basal ATP production rate in EOMA was much higher than that of MAE as reported above, the total ATP balance in EOMA cells was roughly half of that measured in MAE (Fig. 4d). This finding hints toward a very high rate of ATP consumption in EOMA, consistent with its much higher growth rate compared with MAE. NBE, but not weak XRT alone, further depleted ATP levels specifically in EOMA cells. Although ATP levels in EOMA cells were lower than that in MAE cells, the ATP/ADP ratio was comparable. This ratio was markedly decreased in response to NBE. While weak XRT alone did not influence this ratio, together with NBE it further elevated ADP levels in a way that the ratio was further decreased (Fig. 4e, f).

\section{Mitochondrial fission and membrane potential}

Morphological evidences by electron microscopy show more abundance of mitochondria in EOMA compared with MAE (Supplementary Fig. S1). NBE induces dynaminrelated protein 1 (Drp1), a marker of mitochondrial fission 


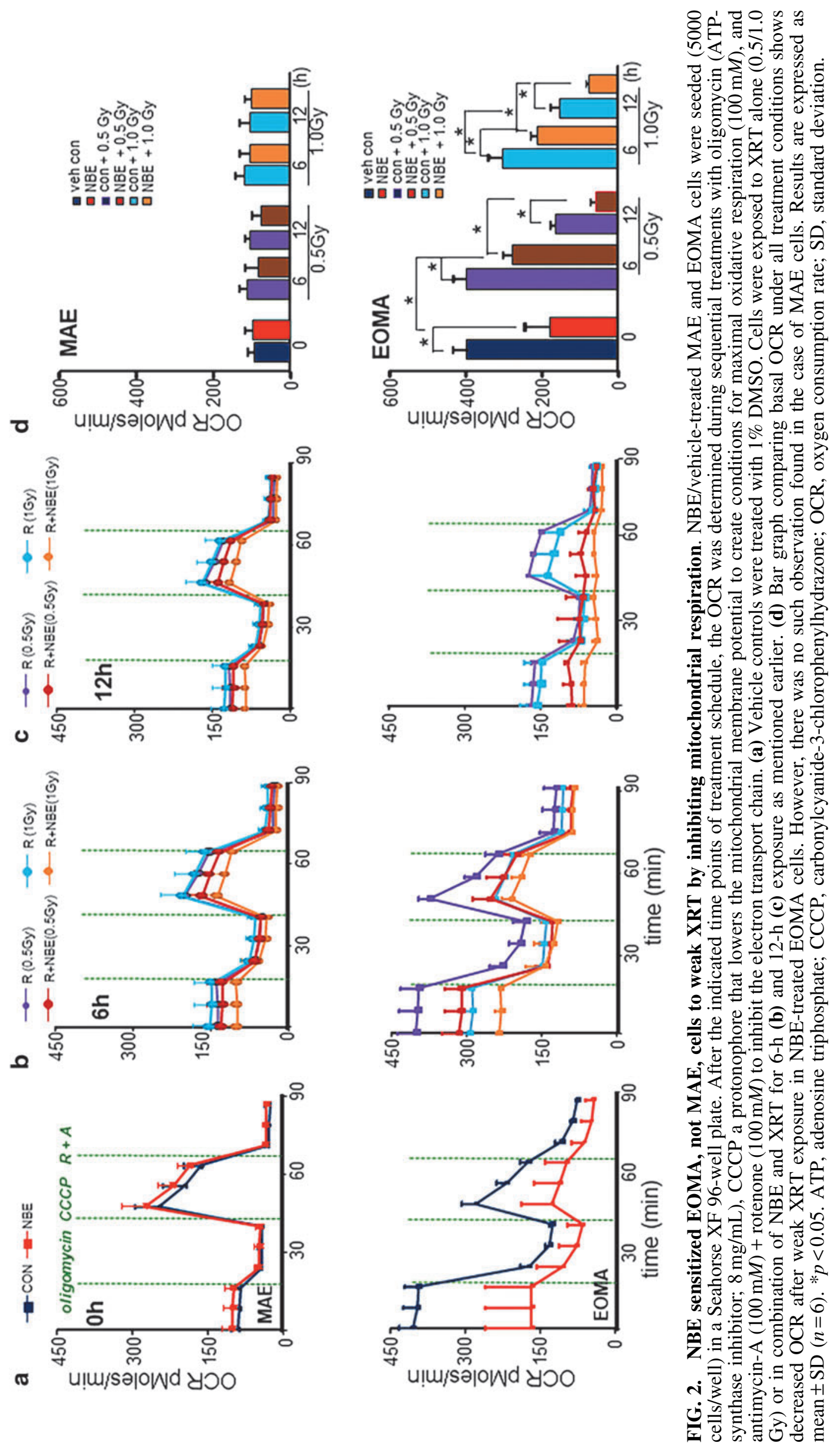


\&
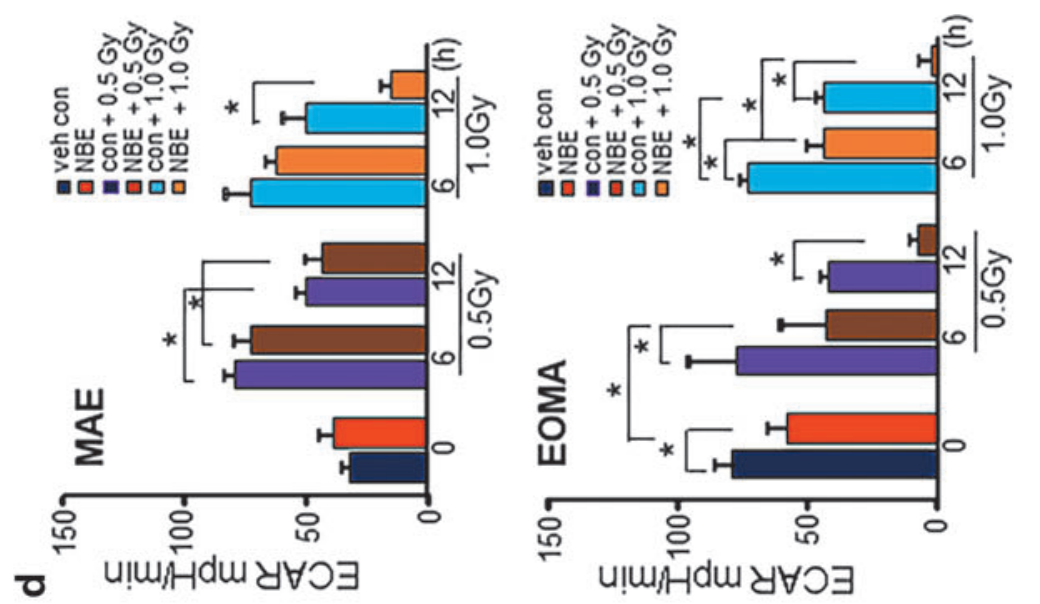

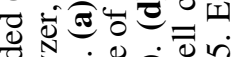

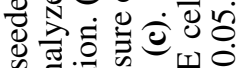
ส

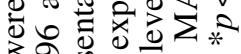

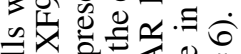

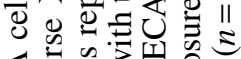

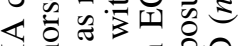

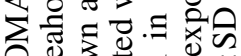

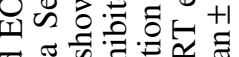

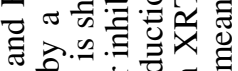
띠월

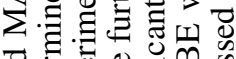
ए

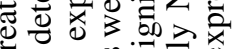
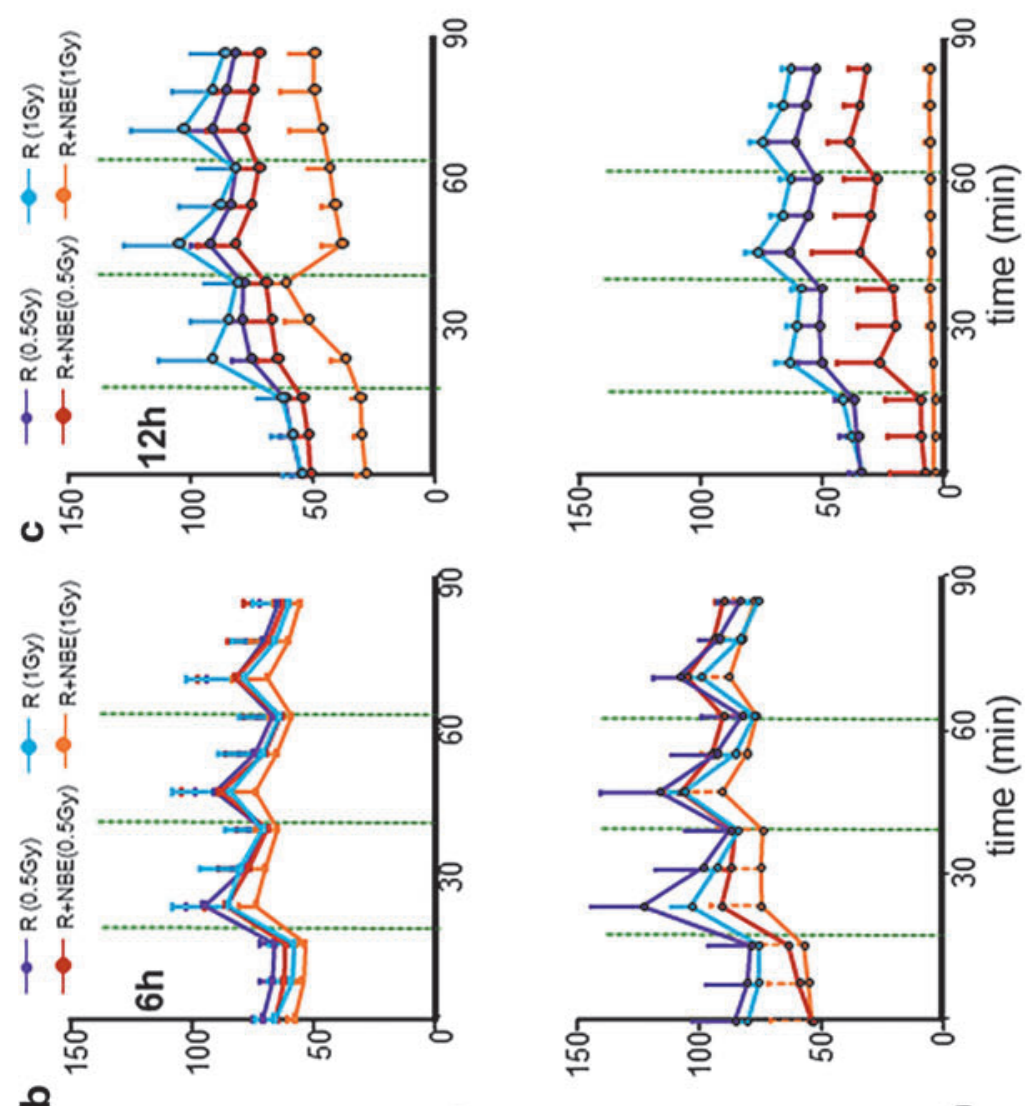

ن

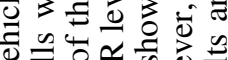

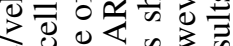
ज्ञ Z $\propto \dot{\sim} \dot{s} \dot{s}$ 날

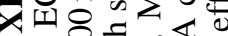

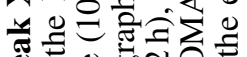
메능

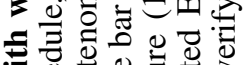

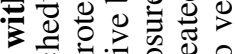

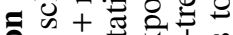
a

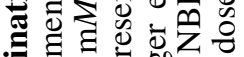

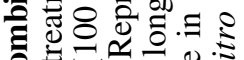
는

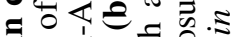

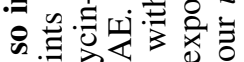

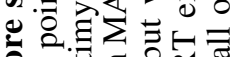
ํ. 킁

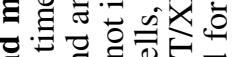
च of

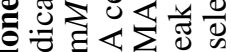
ส. Z

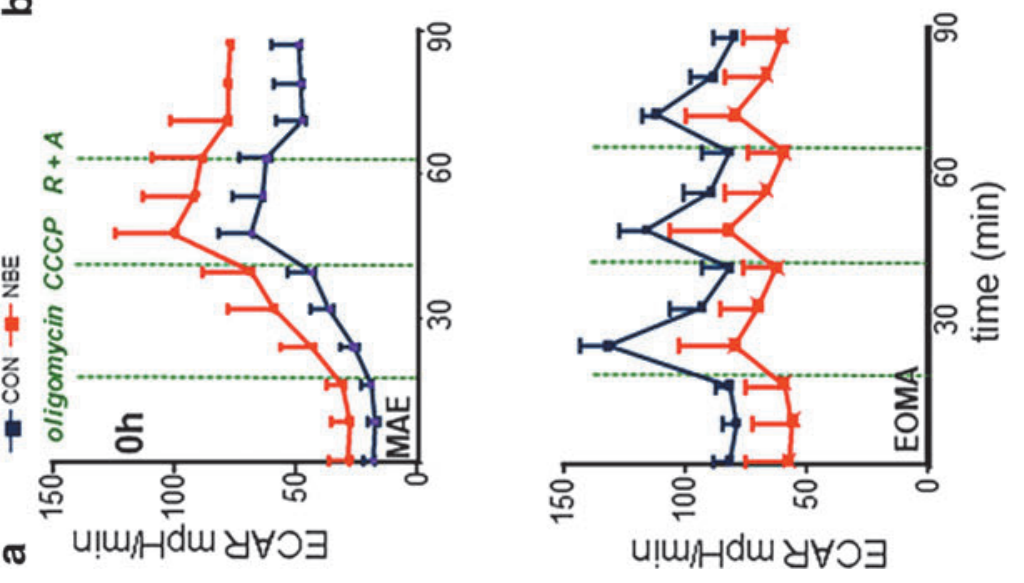

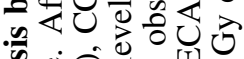

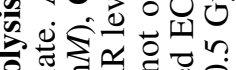

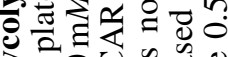

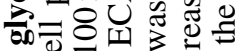

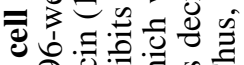
a 0 运

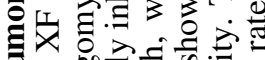

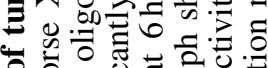

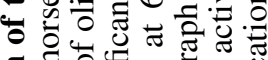
ह क

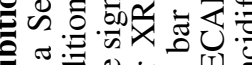

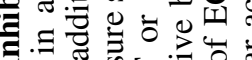
羊西 ம்

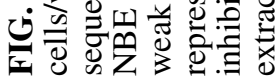


a

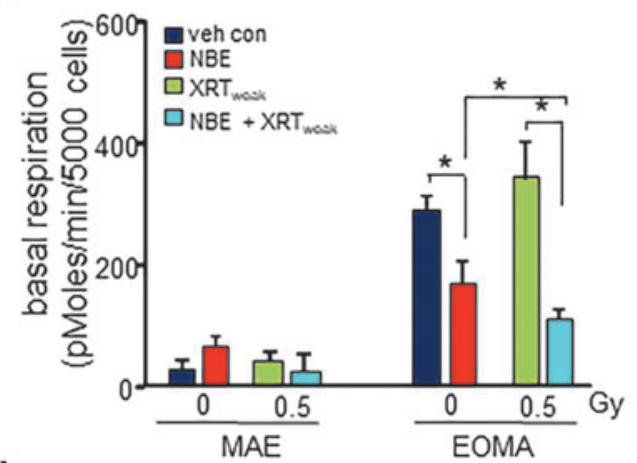

b

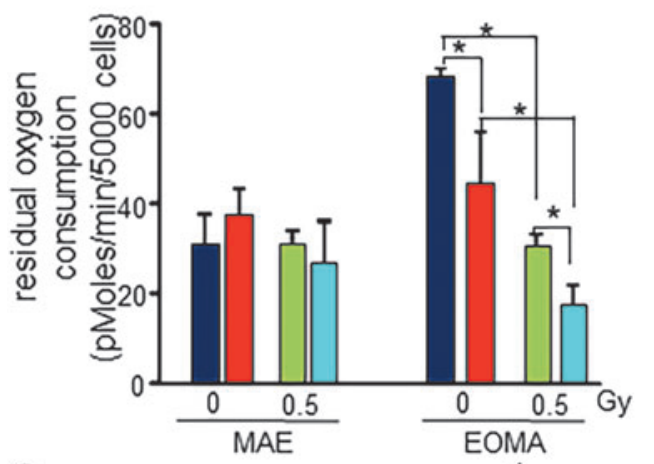

C

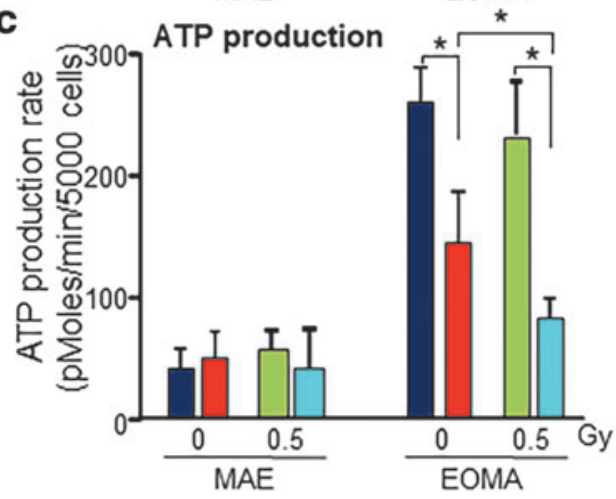

d

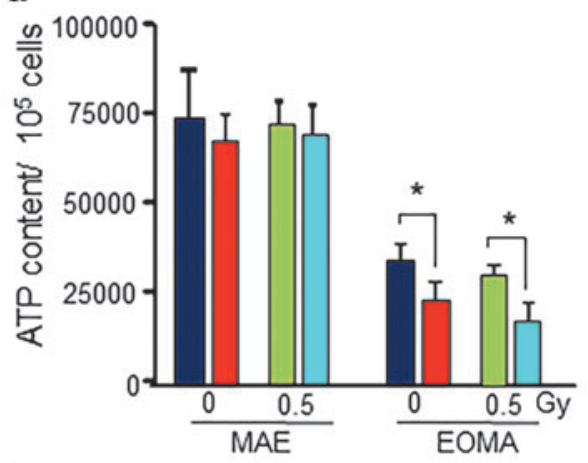

e

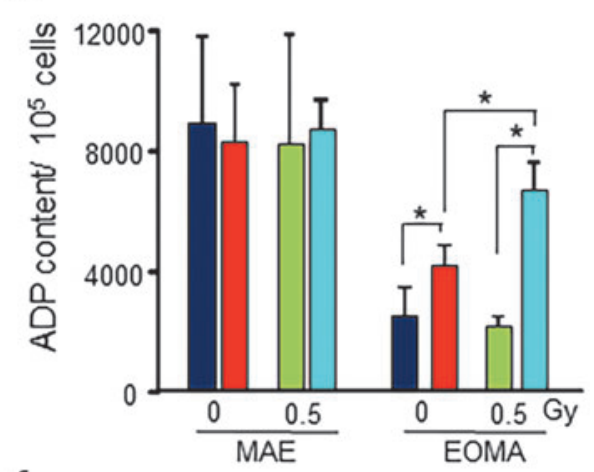

f

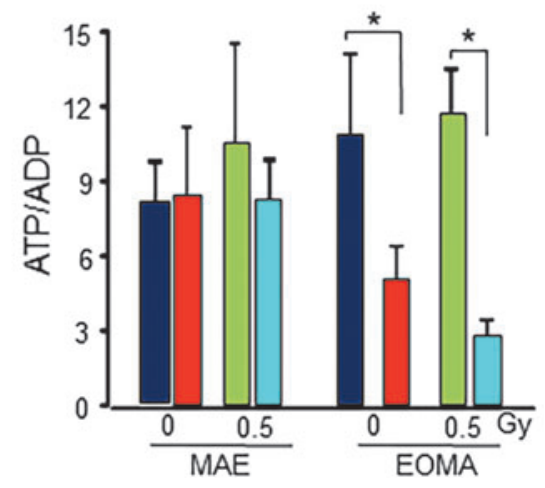

FIG. 4. Blunted cellular respiration and reduced ATP production in EOMA cells. (a) Basal respiration measured from Seahorse XF96 was significantly inhibited with weak XRT in NBE-treated EOMA compared with MAE. (b) The residual oxygen consumption also gets compromised with weak XRT exposure in NBEtreated EOMA cells. No such effects were observed in nontumor forming $\mathrm{MAE}$ cells. (c) Weak XRT exposure causes a decreased rate of ATP production in NBEtreated EOMA cells compared with the no weak XRT group. All calculations were made using the Agilent Seahorse software provided with the instrument. Results are expressed as mean $\pm \mathrm{SD}$ $(n=6)$. (d) Basal cellular ATP, ADP (e), and the ratio (f) were measured in different treatment groups using the EnzyLightTM ADP/ATP ratio assay kit. Results are expressed as mean \pm SD $(n=4) .{ }^{*} p<0.05$. protein, in both MAE and EOMA (Fig. 5a-c and Supplementary Figs. S2 and S3). Recruitment of Drp1 to mitochondrial tubular network was clearly seen in EOMA cells, however, fragmentation was not complete (Supplementary Fig. S2). In the presence of weak XRT, such induction was abrogated in MAE but not in EOMA (Fig. 5b, c, and Supplementary Fig. S2). Mitofusin 2 (Mfn-2), a marker of mitochondrial fusion, was more abundant in EOMA cells compared with MAE (Fig. 5b, d). In MAE, not NBE but weak XRT induced Mfn-2. Such weak XRT-dependent induction was blunted in the presence of NBE (Fig. 5b, d). Increased expression of mitochondrial fission factor (MFF) and simultaneously decreased dynamin-like protein (OPA1) expression indicate fission in response to combined treatment of NBE and weak XRT (Supplementary Fig. S4). Basal mitochondrial copy numbers in EOMA cells were high compared with MAE (Fig. 5e). Exposure to NBE reduced the mitochondrial copy number selectively in EOMA but not in MAE (Fig. 5e). This effect was consistent with exposure to $\mathrm{XRT}_{\text {weak }}$ and combination of $\mathrm{NBE}+\mathrm{XRT}_{\text {weak }}$ (Fig. 5e). In
EOMA cells with higher baseline levels of Mfn-2 compared with MAE, NBE lowered the expression of Mfn-2. Interestingly, in these cells, NBE and weak XRT acted cooperatively in markedly lowering the abundance of Mfn-2 (Fig. 5b, d). Studies testing additional parameters aimed at rigor showed decreased mitochondrial fusion marker protein (OPA1) and increased mitochondrial fission marker protein (MFF) in response to $\mathrm{NBE}$ or $\mathrm{NBE}+\mathrm{XRT}_{\text {weak }}$ combined treatment (Supplementary Fig. S4).

Depolarization of the mitochondrial membrane potential $(\Delta \Psi \mathrm{m})$ is a hallmark of compromised mitochondrial function (65). Flow cytometric analyses of mitochondrial $\Delta \Psi \mathrm{m}$ revealed that EOMA cells demonstrate threefold higher $\Delta \Psi \mathrm{m}$ under basal conditions compared with that in MAE (Fig. 5f-i). In MAE, neither NBE nor weak XRT displayed any effect on $\Delta \Psi \mathrm{m}$. However, in EOMA cells, NBE potently compromised $\Delta \Psi \mathrm{m}$. Such deleterious effect of NBE on mitochondrial function of EOMA cells was markedly enhanced by the combined treatment of weak XRT (Fig. 5f-i). Neither caspase 3 cleavage nor the expression of its upstream 

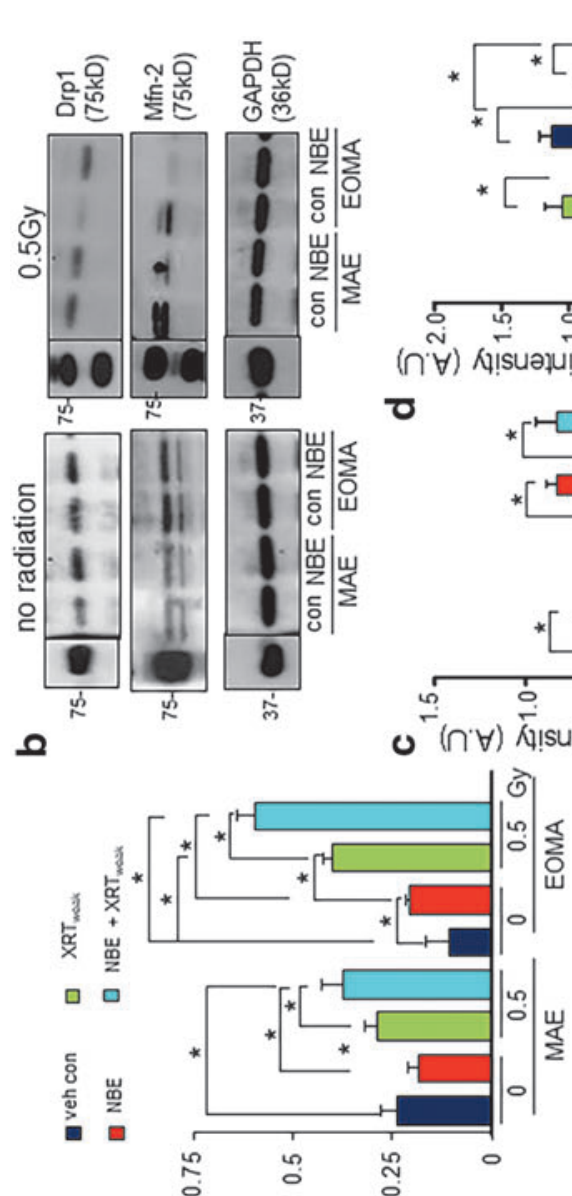

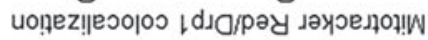

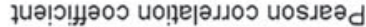
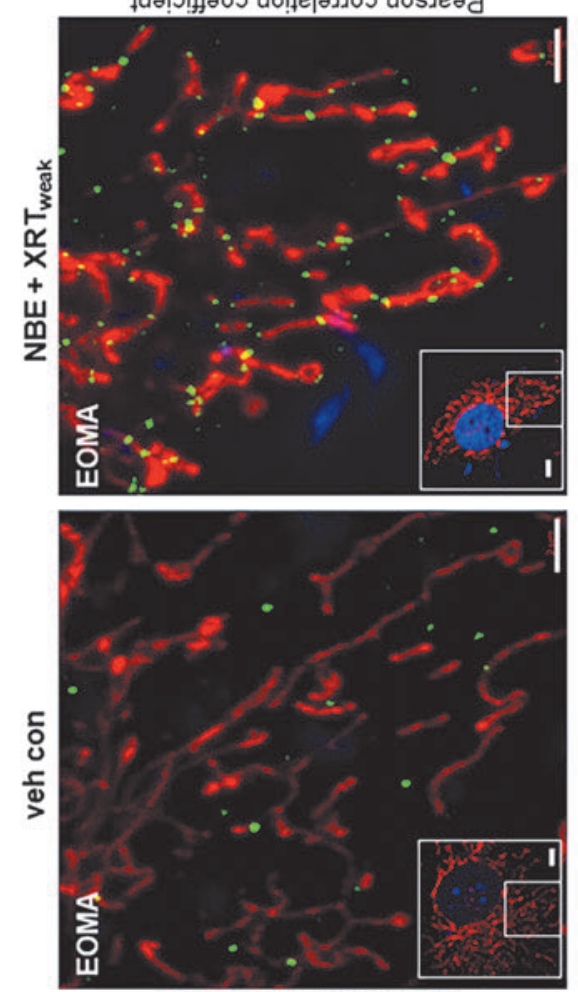

$\boldsymbol{v}$ ठ

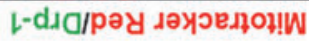

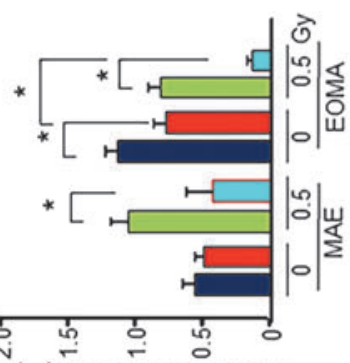

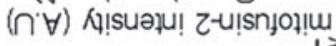

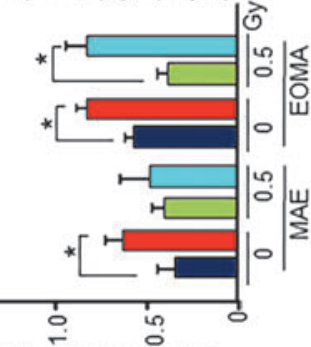

o
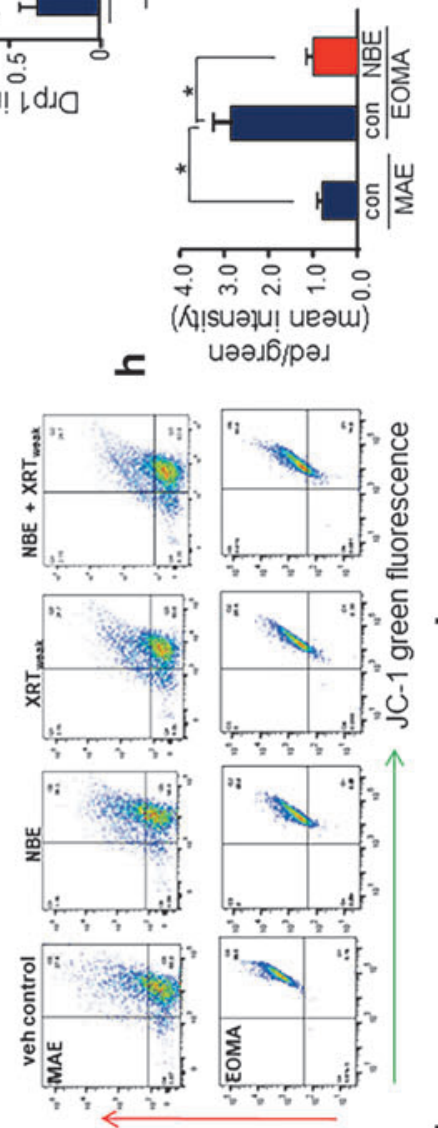

ס
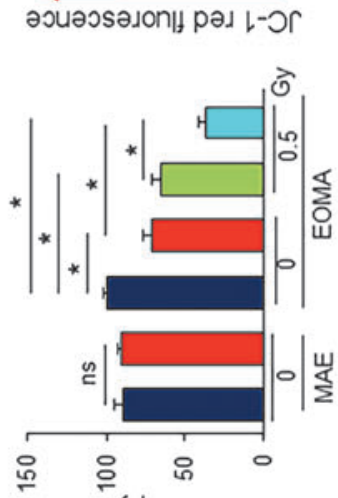

(1) sıaqunu Kdos $\forall$ Naju

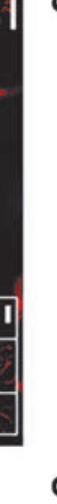

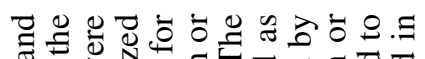

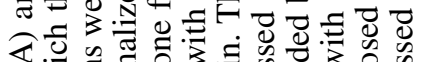
$\varangle \cdot \frac{1}{3} 00$ sos.

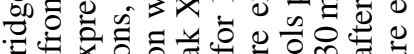
- ख.०

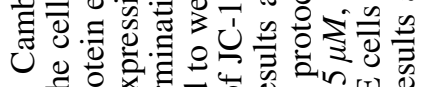
E

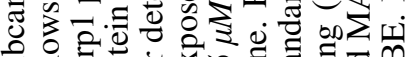

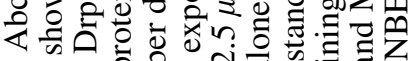
母ัฐँ 곡

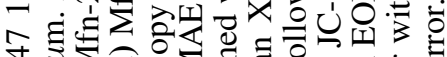

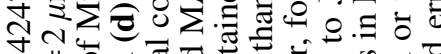
少 ส 0 品 -

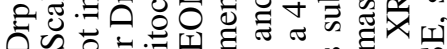
的

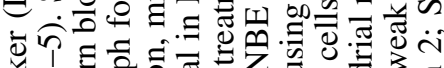

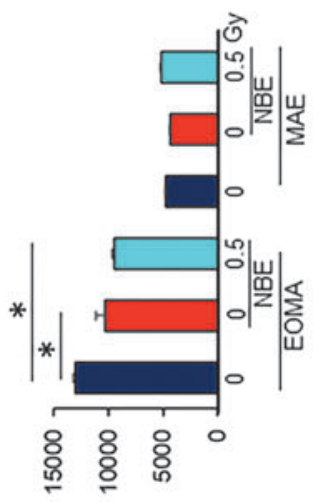

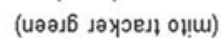
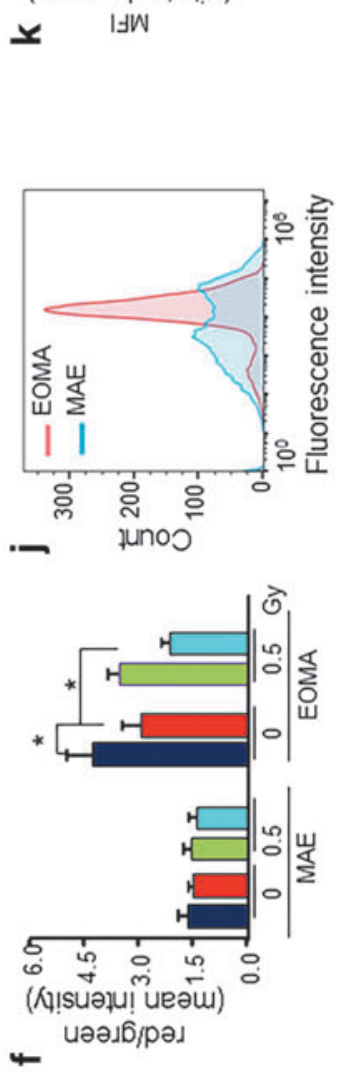

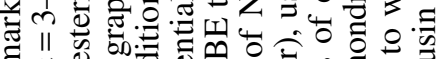

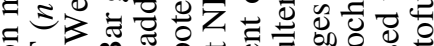

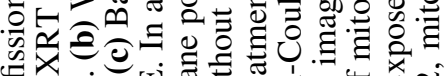
สี

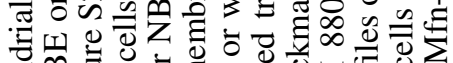

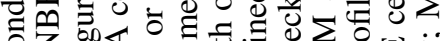

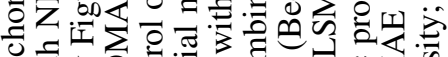

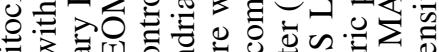

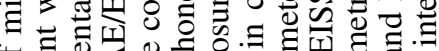

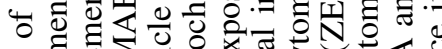

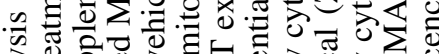

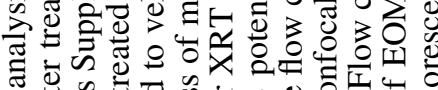

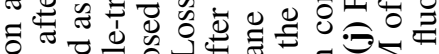

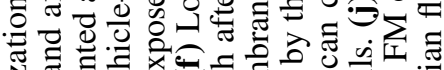
청 司 ฮั 융.

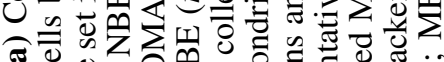

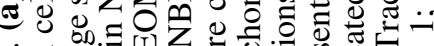

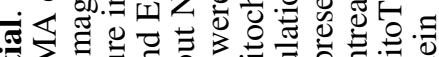

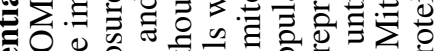

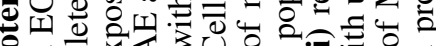

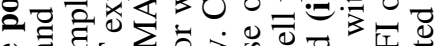
ษ

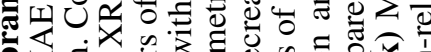

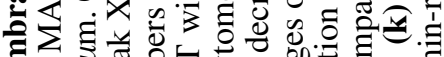

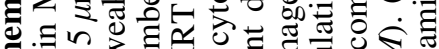

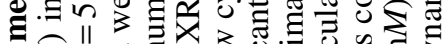

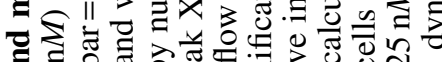
๘

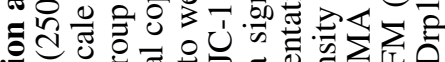
की का.

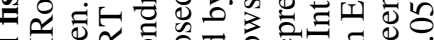

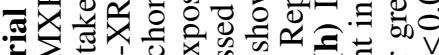

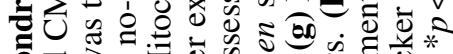

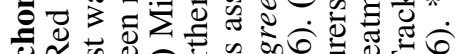

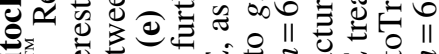

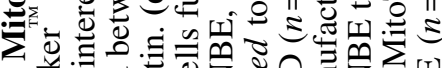

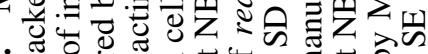

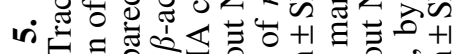

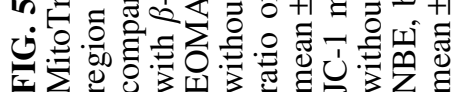


regulator caspase 9 was responsive to a combined treatment with NBE and weak XRT (Supplementary Fig. S3) ruling out the involvement of this particular apoptotic pathway.

\section{Low sirtuin 3 induces death of EOMA cells}

Sirtuin 3 (SIRT3) is a mitochondrial protein that contributes to cell survival by a number of mechanisms, including regulation of mitochondrial dynamics $(3,6,32,43)$. Abundance of SIRT3 protein was markedly lower in EOMA cells compared with that in MAE (Fig. 6a). NBE treatment selectively depleted SIRT3 in EOMA cells, but not that in MAE. In combination with weak XRT, NBE was even more potent in depleting SIRT3 levels in EOMA cells (Fig. 6b). To test the functional significance of such SIRT3 depletion in EOMA cells, pyrroloquinoline quinone (PQQ), a potent inducer of SIRT3, was studied (62). PQQ induced SIRT3 in both cells, but the magnitude of induction was much higher in EOMA cells (Fig. 6c). The $20 \mu M$ dose was chosen for further studies as tangible cytotoxicity was detected with a higher dosage (Fig. 6d). PQQ treatment restored SIRT3 in EOMA cells treated with a combination of NBE and weak XRT (Fig. 6f). Such restoration of SIRT3 abrogated the cytotoxic properties of the combination of NBE and weak XRT (Fig. 6e). These findings support the contention that in EOMA cells, SIRT3 depletion causes cell death. Further evidence to rigorously test that contention, an SIRT3 inhibitor LC-0296 (1), was utilized. LC-0296 potently depleted SIRT3 in EOMA cells (Fig. 6g). Such severe depletion alone caused EOMA cell death in a way that was more severe than the effect of NBE. Taken together, neutralizing SIRT3 is productive in killing EOMA cells. The combination of NBE and weak XRT exploits that avenue of tumor cell death.

\section{AMP-activated protein kinase phosphorylation caused by severe depletion of SIRT3 compromises mitochondrial respiration in EOMA cells}

Induction of SIRT3 by PQQ lowered phospho-AMPK (AMP-activated protein kinase) abundance in both MAE and EOMA cells (Fig. 7a). Inverse relationship between abundance of SIRT3 and phospho-AMPK was also evident in studies comparing MAE with EOMA. In EOMA, low SIRT3 was associated with high phospho-AMPK (Fig. 7b, c). Phospho-AMPK is expressed in a way that is normalized by the overall abundance of AMPK under those conditions. As reported above, NBE treatment compromised mitochondrial function as well as marginally depleted SIRT3 in EOMA cells, yet under these conditions phospho-AMPK remained unaffected. However, under conditions of combination treatment with NBE and weak XRT, severe depletion of SIRT3 (Fig. 6a) elevated phospho-AMPK (Fig. 7b, c). PQQ rescued against loss of mitochondrial respiration in EOMA cells treated with a combination of NBE and weak XRT (Fig. 7d). Consistently, rescue against loss of membrane potential was also evident in response to PQQ treatment (Fig. 7e). These observations argue in favor of a critical role of SIRT3 depletion in causing AMPK phosphorylation followed by mitochondrial dysfunction. Thus, AMPK phosphorylation caused by severe depletion of SIRT3 compromises mitochondrial respiration in EOMA cells.

\section{Improved survival of mice with HE tumor}

To determine the functional impact of combination treatment of NBE and weak XRT, mice with HE (22) in the control arm received hyaluronic acid cream (29) topically and water orally as vehicle control. The three treatment arms included the following: topical and oral NBE treatment also known as the NBE arm; 7.5 Gy XRT over 3 days or weak XRT directed at the tumor in 3 fractionated doses; and a combination of the above two also known as the NBE+weak XRT combination treatment group. Tumor volume and blood velocity were determined by Doppler ultrasound (Fig. 8a). Tumors in all treatment groups were of the same size on day 3 following EOMA cell injection before beginning any of the therapeutic interventions. In the control arm, rapid increase in tumor volume was noted in 10 days. In this group, the first death was also registered on day 10. Both NBE and weak XRT alone significantly limited tumor volume. This effect was most prominent in the combination treatment group (Fig. 8b). As expected, the changes in blood flow velocity showed the same pattern of response as seen with tumor size (Fig. 8c). The tumors were also qualitatively different between treatment groups with control animals having large, firm, slightly spongy blood-filled tumors that had abundant tumor parenchyma and stroma. Irradiated tumors were fluidfilled sacs with minimal tumor stroma and no appreciable parenchyma. NBE-treated mice had small firm tumors with minimal tumor parenchyma and stroma. NBE and XRT had a fluid-filled sac, but no appreciable tumor parenchyma or stroma (Fig. 8d, e). A Kaplan-Meier survival curve was done in a separate cohort of mice, which received the same treatment protocols, and results were consistent with the other in vivo measurements showing a progressive increase in survival from control, NBE only, weak XRT only, and NBE with XRT (Fig. 8f). Thus, both NBE and XRT were able to prolong survival in these mice with the combination of the two treatments being the most effective.

\section{Discussion}

DNA damage of tumor cells caused by ionizing radiation has been a productive therapeutic strategy with over twothirds of all cancer patients dependent on ionizing radiation therapy (10). This approach targets rapidly proliferating tumor cells justifying its wide usage. However, depending on its dosage, adverse side effects caused by radiation therapy are of serious concern (10). Efforts to find conditions under which low radiation exposure may yield maximum benefits are therefore highly valuable. Radiosensitizers and enhancers are therefore of interest. To achieve best health outcomes, lower doses of radiation are often used in combination with conventional chemotherapy. While this is of broad interest, this approach has its own limitations, primary of which is the fact that in general, chemotherapeutic drugs themselves are highly cytotoxic not only for tumor cells but also for other healthy cells of the body. The adverse side effects of both radiation and chemotherapy pose serious threat to the recovery and quality of life of the surviving patient (9). Phytochemicals, such as anthocyanins, are not only safe but often desirable because of their antioxidant and other beneficial properties (20). An exciting development in tumor management is the observation that dietary phytochemicals exhibit strong chemopreventive properties (33). Our previous work 


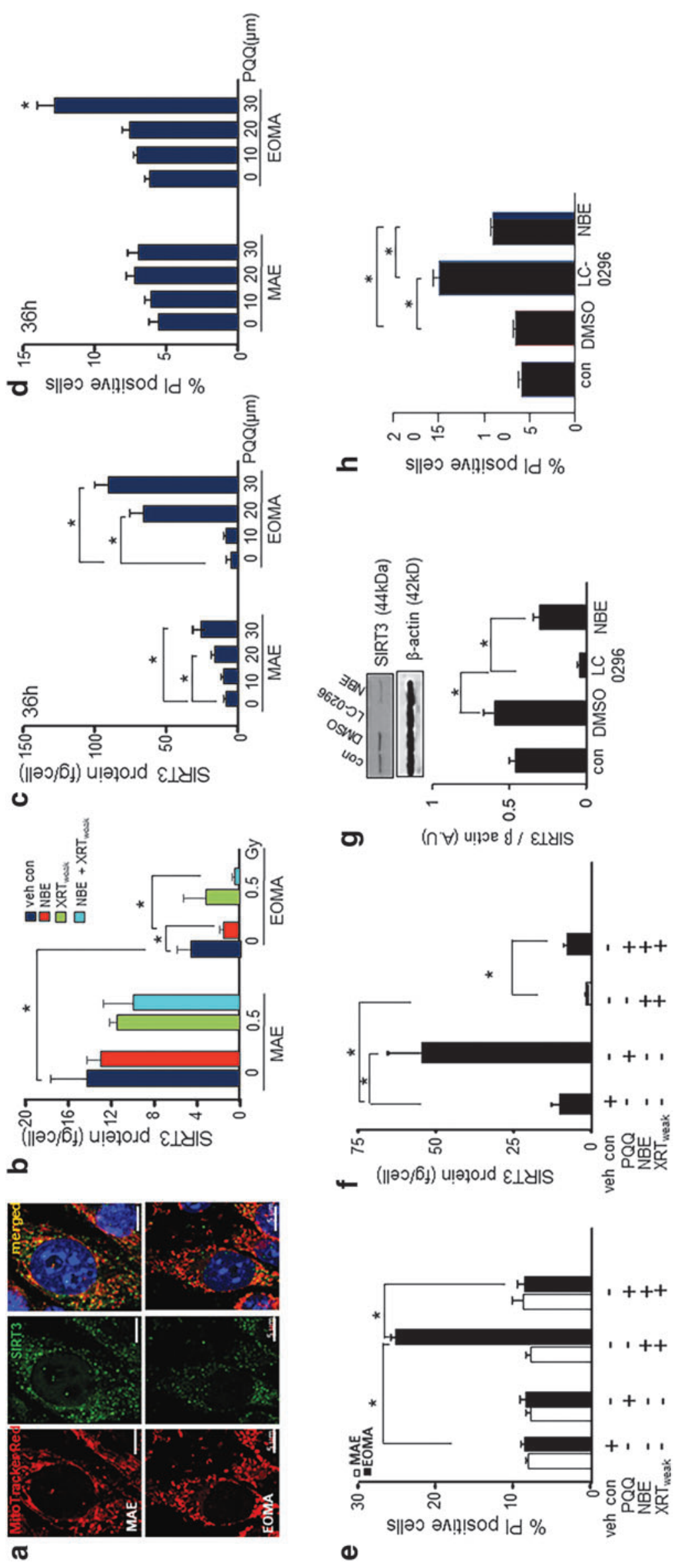

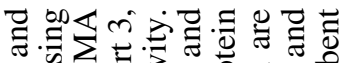

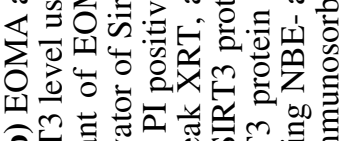
อิ

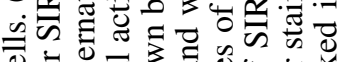

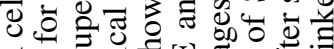

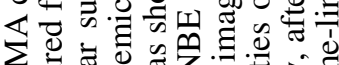

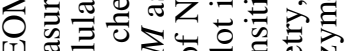
呵 ส

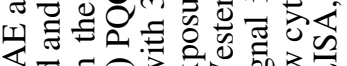

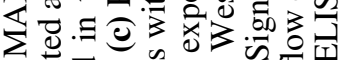

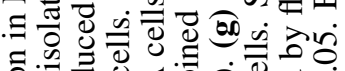

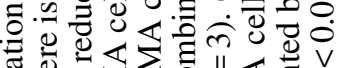

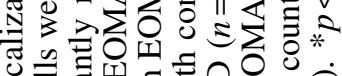

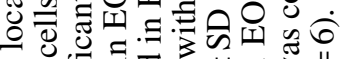

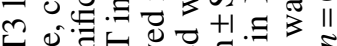

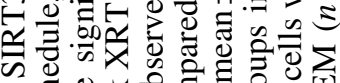
ప टٓ

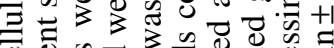

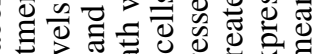

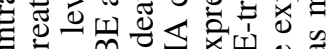

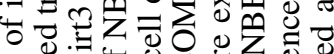

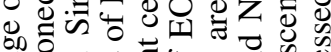
छั:

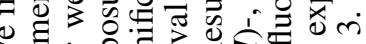

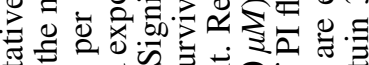

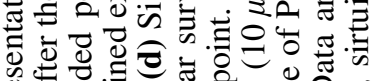

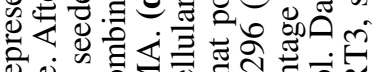
樀

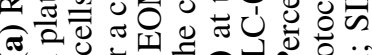

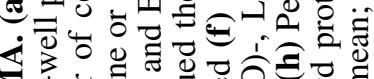
范 근

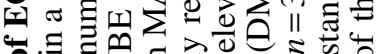
을

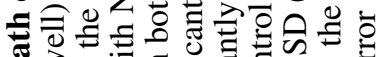

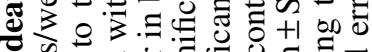
웡

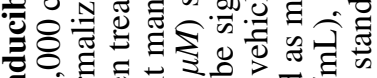

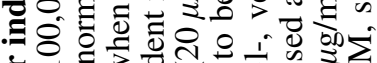

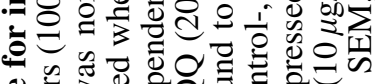

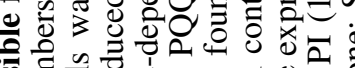

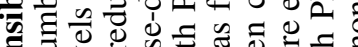

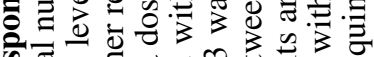

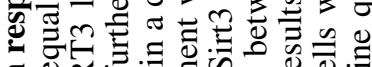

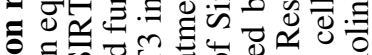

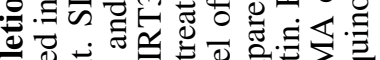

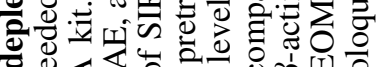
0 近识

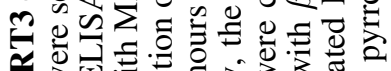

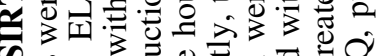

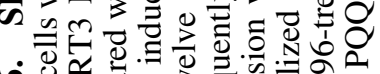

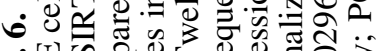

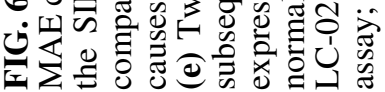




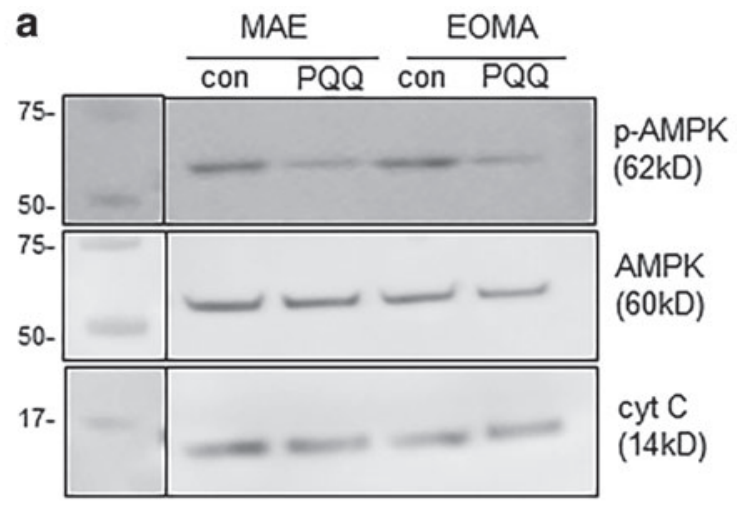

b
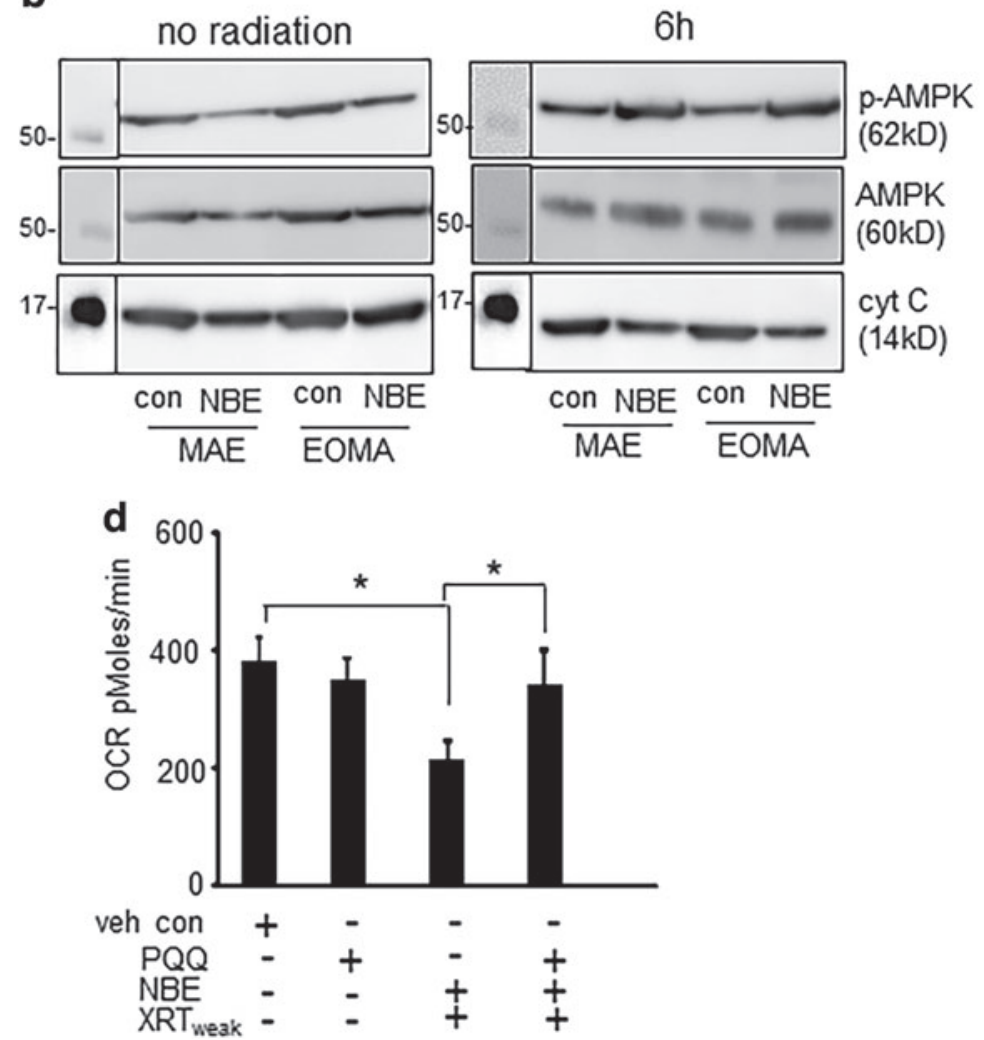
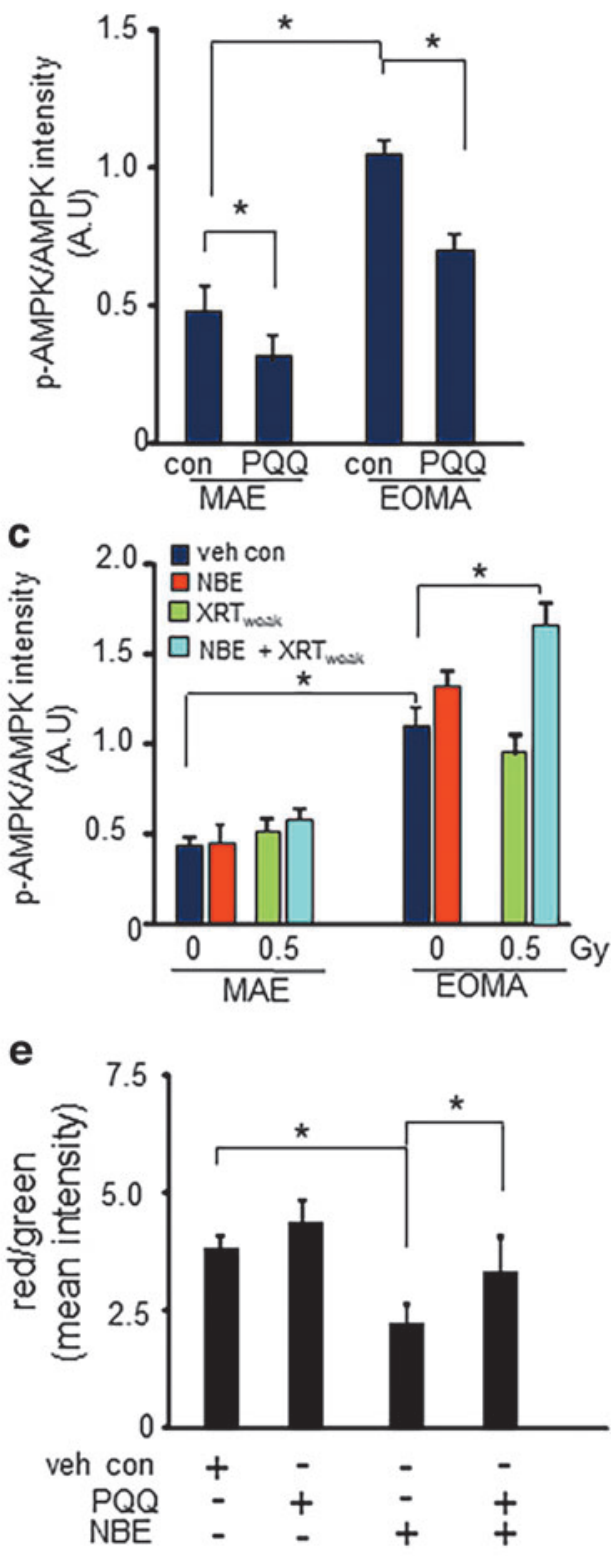

FIG. 7. AMPK phosphorylation caused by severe depletion of SIRT3 compromises mitochondrial respiration in EOMA cells. (a) Western blot shows elevated ratio of phospho-AMPK to AMPK in EOMA cells compared with MAE. PQQ treatment reduces the AMPK activation by inhibiting phosphorylation in both MAE and EOMA cells. (b) Western blot images of phospho-AMPK and AMPK in NBE alone or combined exposure of NBE and weak XRT. (c) Intensity calculation confirms that either NBE alone or in combination with weak XRT increases the phosphorylation of AMPK in EOMA cells. Results are expressed as mean $\pm \mathrm{SD}(n=3)$. (d) OCR data show that pretreatment with PQQ rescued the combined effect of NBE and weak XRT. (e) Intensity calculation of EOMA cells subjected to JC-1 staining with or without NBE or PQQ treatment. Results are expressed as mean \pm SD $(n=7) .{ }^{*} p<0.05$. AMPK, AMP-activated protein kinase.

has demonstrated that a standardized natural edible berry extract (NBE) is capable of prolonging survival of mice affected with HE (11). Thus, for certain tumors, these phytochemicals may have a role beyond chemoprevention where therapeutic benefits may be expected. NBE is now awarded Food and Drug Administration-Investigational New Drug (FDA-IND; 140318), for human testing against infantile hemangioma. As such efforts progress, it becomes critically important to understand the mechanisms of action of NBE in settings involving vascular neoplasms. Furthermore, it becomes important to ask whether NBE may cooperate with ionizing radiation such that best outcomes may be obtained with radiation so weak that it is otherwise ineffective by itself.

Otto Warburg's proposal of the heavy reliance of cancer cells on aerobic glycolysis, as opposed to mitochondrial OXPHOS, for their energy supply has now evolved to recognize that mitochondrial function is essential for tumor survival. Thus, in addition to applying brakes on the cell cycle, strategies to specifically disrupt mitochondrial function of tumor cells are likely to be valuable (55). A combination approach to target cell cycle and mitochondrial function of tumor cells is likely to be productive especially in rapidly growing tumor 


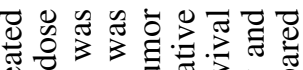

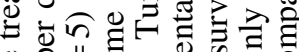
o.

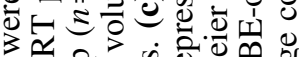

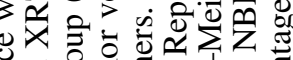

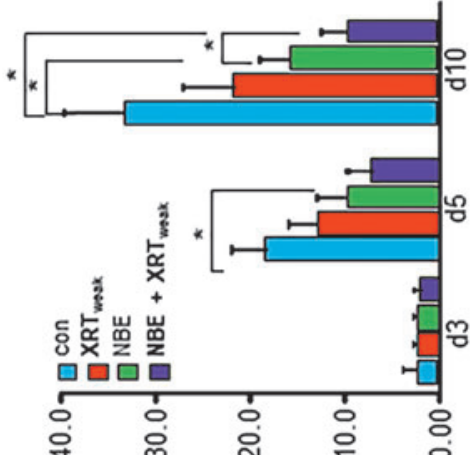

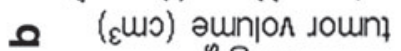
을응
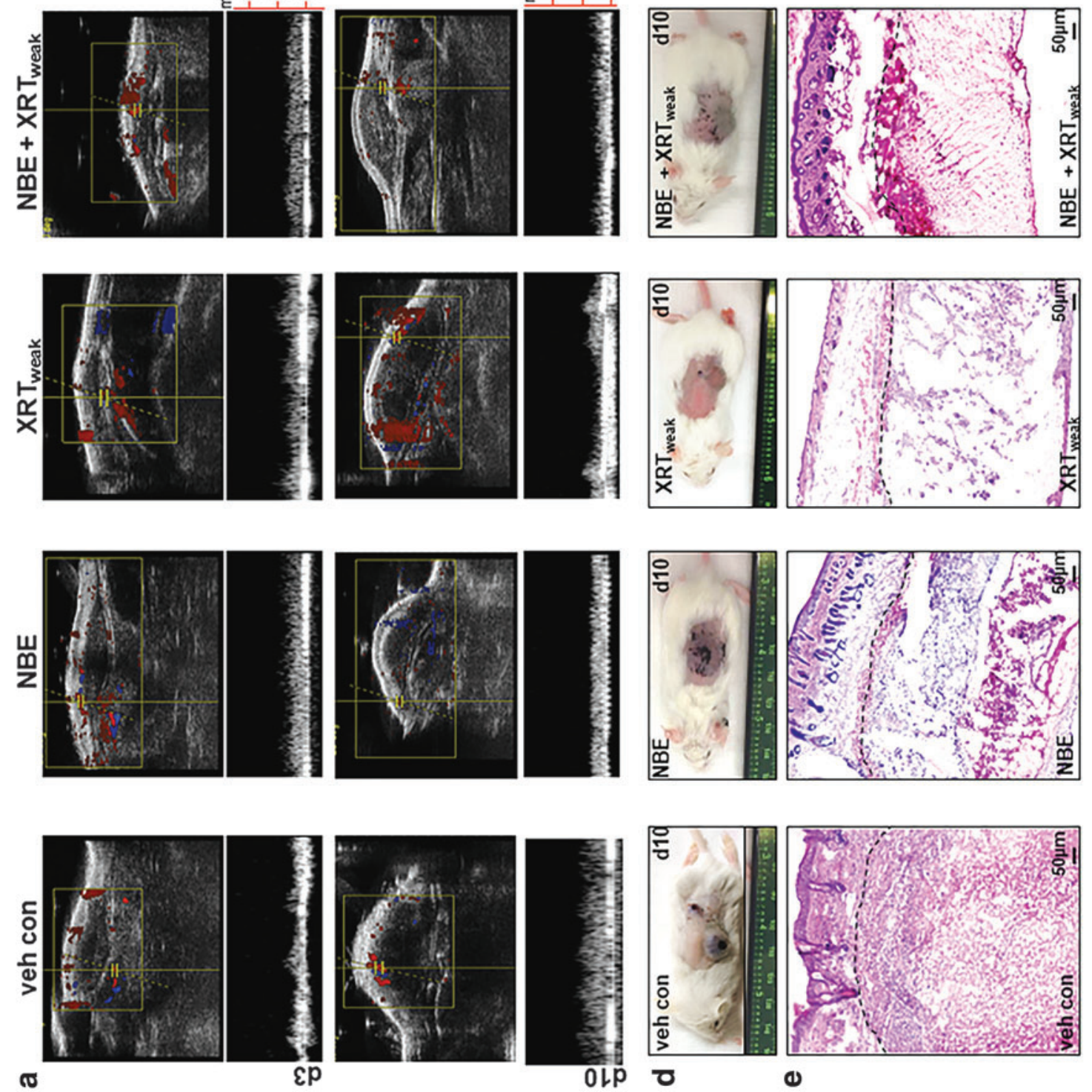

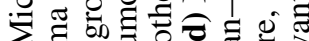

政

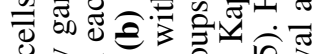

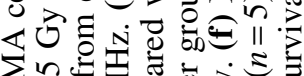
$\sum n=0$

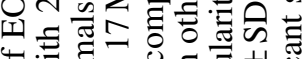

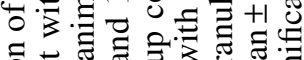

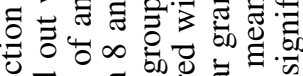
递实 on

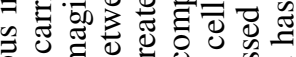
$y .900$

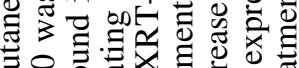

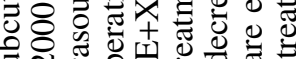
क त

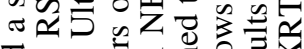

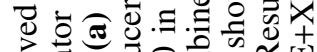

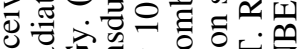

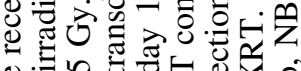
. in $=0$

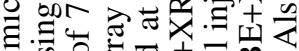
जo a o t ते के

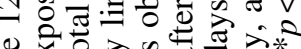
중 훈,

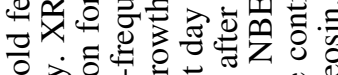

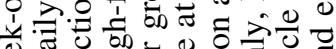

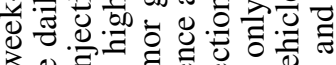

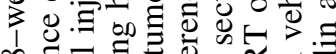

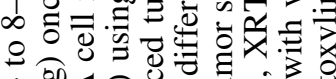

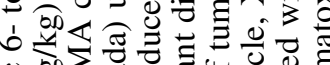

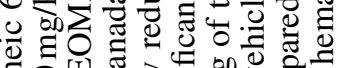
呵

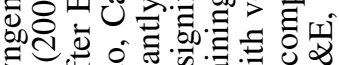

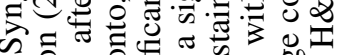

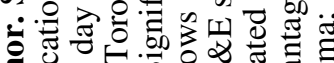

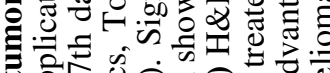

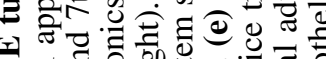

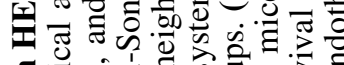

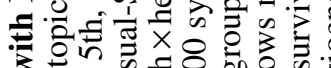

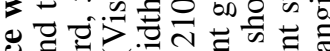

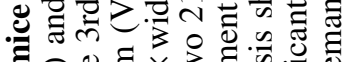
四 항ㅎㅁ

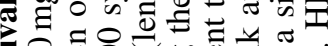

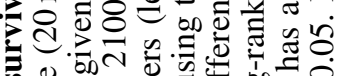
के

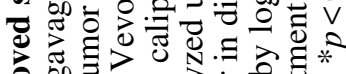

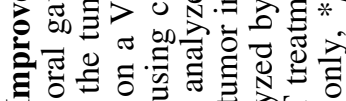

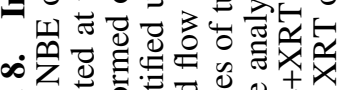

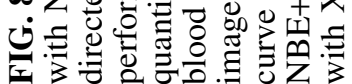


cells heavily relying on their mitochondrial function for their sustenance. Bioenergetic alterations in tumor cells are diverse. While some are more glycolytic, others rely on OXPHOS of the mitochondria (55). This work presents first evidence on the strikingly hyperactive mitochondria in EOMA cells. In this work, a combination of weak XRT (0.5 Gy) and NBE was most effective in killing EOMA cells, when only doses that were safe for healthy MAE cells were considered. While NBE alone was effective in causing cell death, the effect was significantly enhanced by cotreatment with weak XRT. These observations provided the first cues for the design of in vivo rescue experiments. Interestingly, mitochondrial OCR of EOMA was threefold more than that of MAE, suggesting heavy reliance of these cells on their mitochondrial function. At dosages used, both NBE and weak XRT were safe for MAE. Yet, NBE caused marked lowering of mitochondrial respiration. These results are consistent with our earlier findings (4). Weak XRT was also effective in blunting mitochondrial respiration. In combination, NBE and weak XRT severely attenuated mitochondrial function. This work is the first to report on such cooperative effects of a phytochemical and weak XRT on selectively limiting tumor mitochondrial functions in a way that favorably affects tumor outcomes in vivo. Glycolysis contributes to tumor cell metabolism. Compared with that of MAE cells, the rate of glycolysis in EOMA was twofold higher. Specifically, in EOMA cells, NBE marginally inhibited glycolysis. Weak XRT induced glycolysis in healthy MAE cells. This observation is consistent with studies testing higher doses of radiation to demonstrate an acute phase of induction of glycolysis (64). In EOMA cells, however, with already high baseline rates of glycolysis, weak XRT inhibited glycolysis in the longer term. Inhibition of glycolysis in tumor cells by ionizing radiation has been reported (19). High ATP turnover, high proton leak, or elevated nonmitochondrial oxygen consumption may account for high basal respiration of the cell (27). Compared with MAE cells, EOMA cells showed sixfold higher basal respiration. In the hepatocytes and cardiomyocytes, nonmitochondrial oxygen consumption constitutes $\sim 40 \%$ of basal OCR, and in endothelial cells $\sim 15 \%$ (27). In EOMA, nonmitochondrial oxygen consumption accounted for $15 \%$ of its basal respiration. Such oxygen consumption is known to contribute to reactive oxygen species (ROS) formation in the cell. EOMA cells, rich in ROS, rely on these oxygen derivatives for their growth (12). NBE inhibited both basal and nonmitochondrial respiration of EOMA cells, while not affecting MAE cells. While weak XRT had no effect on basal respiration, it inhibited nonmitochondrial respiration selectively in EOMA. A combination of NBE and weak XRT did not have any effect on healthy MAE cells. However, it markedly inhibited both basal and nonmitochondrial respiration in EOMA cells. While the ATP production rate of EOMA cells was fivefold higher than in MAE cells, the ATP balance in these cells was roughly half indicating rapid ATP turnover consistent with its high rate of tumor growth. The ATP/ADP ratio was comparable between MAE and EOMA. NBE lowered the rate of ATP production, depleted ATP content, elevated ADP, and lowered ATP/ADP ratio. Such effect was selectively observed in EOMA, while MAE cells remained unaffected. While weak XRT did not influence these parameters on its own in either cell type, it potentiated the effect of NBE in lowering the production rate of ATP and elevation of ADP.
Polyphenol-induced loss of tumor mitochondrial function has been recognized as a physicochemical process that cancer cells cannot develop resistance against gene mutation (39, 48). Anthocyanins, rich in berries, are also known to influence mitochondrial dynamics (39). NBE elevated Drp1 in both MAE and in EOMA, with the distinct contrast that in MAE such increase in fission was not associated with cytotoxicity, while in EOMA it was. With respect to the interpretation of significance of such findings, it is important to put into context changes in mitochondrial fusion under the same conditions. In MAE, NBE did not influence Mfn-2. Thus, increased fission in the context of unchanged fusion had no influence on cell viability. In contrast, in EOMA cells, induction of fission was coupled with significant lowering on fusion resulting in loss of cell viability. Thus, unopposed fission killed EOMA cells. Of outstanding interest was the observation that weak XRT did not influence mitochondrial dynamics in both MAE and EOMA. However, selectively in EOMA, weak XRT markedly potentiated the inhibitory effect of NBE on fusion. Such synergistic effect is likely to explain the potentiation of EOMA cell death in response to a combination treatment with NBE followed by weak XRT. Fusion of the outer mitochondrial membrane occurs when cells are forced to rely on OXPHOS by withdrawing glucose as a carbon source, thus maximizing OXPHOS by stimulating complementation among mitochondria (35). Impaired fusion and lowered $\Delta \Psi \mathrm{m}$ favors mitochondrial fragmentation that is often accompanied by bioenergetic defects, as has been evident in this work in response to combination treatment (35, 49). SIRT3 has been described as a mitochondria-localized tumor suppressor (30). SIRT3 helps mount mitochondrial adaptive response to stress, such as metabolic reprogramming and antioxidant defense mechanisms. It has thus been recognized that targeting SIRT3 holds promise in tumor management (14). Baseline SIRT3 levels in EOMA cells were observed to be one-third of that observed in nontumor MAE cells. In EOMA, low SIRT3 was associated with high phosphorylated AMPK, a key signaling partner of SIRT3 (34). Phosphorylation of AMPK is known to be associated with depleted cellular energy levels as was evident in this work (31). At doses used, both NBE and weak XRT did not influence SIRT3 levels in MAE cells. Yet, NBE was effective in lowering SIRT3 levels in EOMA from low to deficient. Weak XRT alone had no effect on SIRT3 in EOMA. However, it markedly potentiated the lowering effects of NBE. Plant polyphenols are known to function as natural sirtuin inhibitors (26). Downregulation of SIRT3 is of interest in managing human breast and gastric cancer $(17,61)$. This work presents first evidence that NBE and weak XRT may work synergistically to downregulate SIRT3 in vascular tumor cells. The extraordinary significance of such downregulation with respect to tumor outcomes was highlighted in experiments where the therapeutic effects of such combination therapy were completely abrogated under conditions of pharmacological induction of SIRT3 by PQQ.

Studies on cell biology, as discussed above, lead to the notion that a combination of NBE and weak XRT would be effective in improving health outcomes of HE. Several previous studies have demonstrated that mice suffering from $\mathrm{HE}$ survive roughly for 2 weeks $(11,21)$. Consistent survival outcomes were observed in this study. Combination therapy with NBE and weak XRT significantly extended the life span 
to over 6 weeks. NBE alone also improved survival. However, weak XRT alone did not. Taken together, this work presents maiden evidence demonstrating the cooperative action of NBE and weak XRT in managing HE. The mechanism of action of this combination therapy includes selective targeting of mitochondrial dynamics and function of tumor cells. Mitochondria-targeted antitumor therapies are likely to be productive in the management of $\mathrm{HE}$.

\section{Materials and Methods}

\section{Cell culture}

EOMA and MAE cells were maintained under the same conditions as previously described $(12,23)$. In brief, cells were maintained in Dulbecco's modified Eagle's medium (DMEM) supplemented with $10 \%$ fetal bovine serum and $1 \%$ penicillin/streptomycin (complete media), and incubated at $37^{\circ} \mathrm{C}$ and $5 \% \mathrm{CO}_{2}$. NBE $(200 \mu \mathrm{g} / \mathrm{mL})$ and vehicle control (1\% dimethyl sulfoxide [DMSO]) were carried out in cell culture media for $24 \mathrm{~h}$ before initiating experiments. NBE is a proprietary mix of powdered berry extracts (PediaBerry ${ }^{\mathrm{TM}}$; NutrimiR, Columbus, $\mathrm{OH})$.

\section{Extracellular flux and OCR (Seahorse ${ }^{\mathrm{TM}}$ ) assays}

OCR and ECAR measurements were performed using a Seahorse Bioscience XF-96 instrument as described previously $(16,45)$. In brief, a day before the experiment, the sensor cartridge was hydrated overnight using the calibration buffer supplied by the manufacturer (Seahorse Biosciences, Santa Clara, CA). The NBE-treated cells were seeded in the 96-well microplate in complete DMEM with $4.5 \mathrm{~g} / \mathrm{L}$ of glucose. On the day of the experiment, the cells were washed with calibration buffer twice and incubated with glucose-free Seahorse XF base medium supplemented with $25 \mathrm{mM}$ of glucose for $1 \mathrm{~h}$ at $37^{\circ} \mathrm{C}$ in a $\mathrm{CO}_{2}$-free incubator. The injection ports of the sensors were filled with $20 \mu \mathrm{L}$ of treatment or vehicle in buffer. The sensor was then placed into the XF-96 instrument and calibrated. After calibration, the calibration fluid plate was replaced with the cell plate. The measurement cycle consisted of a 2-min mix, 1-min wait, and a 2-min measurement. Four basal rate measurements were followed by sequential addition of oligomycin $(8 \mu \mathrm{g} / \mathrm{mL})$, carbonylcyanide3-chlorophenylhydrazone (CCCP, $100 \mu M)$, rotenone $(100 \mu M)$, and antimycin A $(100 \mu M)$ prepared in glucose-free Seahorse XF base medium. Each injection was followed by four measurement cycles. The consumption rates were calculated from the continuous average slope of the decreased $\mathrm{O}_{2}$ using a compartmentalization model (36). For any one treatment, the rates from 10 wells were used. Rates for the wells were normalized for protein content.

\section{Determination of cell viability}

Cell viability was measured by leakage of LDH from cells to media using the in vitro toxicology assay kit from Sigma Chemical (St Louis, MO) as described (12). Cell viability was also analyzed by the PI exclusion test. Cells were incubated with PI $(2.5 \mathrm{mM})$ in phosphate-buffered saline for $15 \mathrm{~min}$ at $37^{\circ} \mathrm{C}$ and with $5 \% \mathrm{CO}_{2}$. Fluorescence intensity was determined by FACS using PI staining in the FL2 region using an Accuri C6 Flow Cytometer (Accuri, Ann Arbor, MI) at 530-nm excitation with a gated sample size of 10,000 cells $(12,41)$.

\section{Measurement of $\Delta \Psi \mathrm{m}$}

$\Delta \Psi \mathrm{m}$ changes were analyzed using the lipophilic cationic dye JC-1 (MitoProbe JC-1 Assay Kit for Flow Cytometry; Life Technologies) as per the manufacturer's instruction by flow cytometer as reported previously (8).

\section{Determination of ADP/ATP levels}

Changes in the ADP/ATP ratio were measured using bioluminescent assay (EnzyLightTM ADP/ATP Ratio Assay Kit; BioAssay Systems) as described previously (42). The ATP content in each sample was corrected for the protein concentration that was determined with the bicinchoninic acid (BCA) protein assay (Pierce).

\section{Western blot}

Immunoblotting was performed using cell lysates, and the protein concentration determined using a BCA protein assay. Samples (15-20 $\mu \mathrm{g}$ of protein/lane) were separated using 4\%-12\% sodium dodecyl sulfate/polyacrylamide gel electrophoresis and probed with the rabbit monoclonal anti-Mfn2 antibody (1:1000 dilution, Cat. No. ab124773; Abcam, Cambridge, MA), rabbit monoclonal anti-Drp1 antibody (1:1000 dilution, Cat. No. ab184247; Abcam), anti-OPA1 antibody (1:1000 dilution, Cat. No. 1284B; Novus Biologicals, Centennial, CO), anti-MFF antibody (1:500 dilution, Cat. No. ab129075; Abcam), anti-phos-AMPK (1:1000 dilution, Cat. No. PA5-17831; Thermo Fisher Scientific, Grand Island, NY), anti-AMPK (1:1000 dilution, Cat. No. NB100239; Novus Biologicals, Littleton, CO), rabbit polyclonal anti-SIRT3 antibody (1:100 dilution, Cat. No. ab86671; Abcam), and anti-mouse $\beta$-actin (1:10,000 dilution, Cat. No. A5441, Lot No. 055K4854; Sigma Chemical). Bands were visualized using the horseradish peroxidase-conjugated donkey anti-rabbit-IgG (1:2000, Cat. No. NA934V, Lot No. 9583369; Amersham Biosciences, Piscataway, NJ) and antimouse-IgG (1:2000, Cat. No. NA931V, Lot No. 6652622; Amersham Biosciences) $(11,12,24)$, and the enhanced chemiluminescence assay (Amersham Biosciences) according to the manufacturer's instructions. Pixel densitometry for individual bands was done using ImageJ software.

\section{SIRT3 enzyme-linked immunosorbent assay}

Cells were seeded in 12 -well plates at $0.1 \times 10^{6}$ cells/well. SIRT3 enzyme-linked immunosorbent assay (ELISA) was performed on cell pellets by using the SIRT 3 ELISA kit (Cat. No. MBS2023250; MY Biosciences, San Diego, CA) according to the manufacturer's instructions. BCA protein assay was performed on an aliquot of all tested samples and the results standardized per milligram of protein.

\section{Transmission electron microscopy}

Transmission electron microscopy was done as per our previous report (57). Briefly, primary fixation of the cells was done with $3 \%$ glutaraldehyde in $0.1 \mathrm{M}$ phosphate buffer. After rinsing in buffer, postfixation followed for $1 \mathrm{~h}$ with $1 \%$ osmium tetroxide in $0.1 \mathrm{M}$ phosphate buffer. After dehydration using ethyl alcohol and acetone as an intermediate solvent, cells were infiltrated over the weekend in $1 / 2$ acetone and $1 / 2$ embedding media. They were then embedded in fresh resin (Embed 812; 
Electron Microscopy Sciences, Hatfield, PA). Following polymerization overnight at $60^{\circ} \mathrm{C}$, the blocks were then ready to section. All steps were done in small centrifuge tubes, including embedding. Thin sections were cut $(80-90 \mathrm{~nm})$, stained with UA replacement stain (Electron Microscopy Sciences), and then viewed on a Tecnai Spirit (Thermo Fisher, Hillsboro, OR). Digital images were taken with an AMT (Advanced Microscopy Techniques, Danvers, MA) CCD camera.

\section{Ultrasound imaging}

A high-frequency, high-resolution ultrasound imaging system (Vevo 2100; Visual-Sonics, Toronto, Canada) with an MS 550D (22-55 MHz) linear array transducer was used as described previously $(18,45,46)$. Mice were anesthetized using a mixture of $1.5 \%$ isoflurane inhalant anesthesia with 95\% oxygen, and animals were placed in the supine position on a moveable heated stage maintained at $37^{\circ} \mathrm{C}$. Hair was removed from tumors using a depilatory cream. Ultrasound scan transmission gel was applied to the tumor surface and the probe was positioned on a fixed stand perpendicular to the stage. The image resolution was maintained at $100 \mu \mathrm{m}$ laterally and $50 \mu \mathrm{m}$ axially. B-mode mapping was performed until clearly visualizing the tumor anatomical location. Each targeted tumor was visualized in two-dimensional (2D) imaging for the visualization of the tumor. Fine position adjustment was done for the highest signal intensity with continuous adjustment of contrast dynamic range and gain until the minimal noise appeared in the background. For tumor volume measurement, different locations on the tumor were set up with the transducer and B-mode recordings were made with the ultrasound beam perpendicular to the targeted tumor. Several recordings (cine-loops), each up to $5 \mathrm{~s}$, of B-mode were saved. Color Doppler flowmetry of tumor vasculature and tumor feeder vessel flow were also recorded using color Doppler flow imaging mode in 2D mode.

The tumor volume was measured during postprocessing. VevoLab software (Visual-Sonics) was used to compile each 2D image slice with other acquired slices and traced the area and depth of the wound resulting into a volumetric data. From the real-time B-mode observation, the framewise tracing of tumor edge borders was performed.

\section{In vitro $X R T$}

Cells were pretreated with NBE $(200 \mu \mathrm{g} / \mathrm{mL})$ or vehicle (1\% DMSO) for $24 \mathrm{~h}$. For the XRT exposure experiments, one multiwell plate was used as an unexposed control and other plates were exposed to either 0.5 (weak) or 1 Gy of XRT using an XRT unit (Precision, North Branford, CT) $(28,54)$. Six or $12 \mathrm{~h}$ after exposure with XRT, cells were collected for further analysis. Here NBE-treated nonirradiated cells were maintained with the exact time points matched with NBE exposure of irradiated cells.

\section{In vivo weak $X R T$}

All animal protocols were approved by the Institutional Animal Care and Use Committee (IACUC) of the Ohio State University (Columbus, $\mathrm{OH}$ ). Mice were maintained under standard conditions at $22^{\circ} \mathrm{C}+2^{\circ} \mathrm{C}$ with $12: 12$ dark: light cycles with access to food and water ad libitum. 129P/3 mice (6-8 weeks, female; Jackson Laboratories, Bar Harbor, ME;
Indianapolis, IN) were subcutaneously injected with vehicle, EOMA cells, as previously described (12). Mice were treated with oral gavaging of NBE $(20 \mathrm{mg} / \mathrm{kg})$ and topical application $(200 \mathrm{mg} / \mathrm{kg})$ once daily (11). Mice were radiated with $2.5 \mathrm{~Gy}$ gamma XRT per dose directed at the tumor given on the $3 \mathrm{rd}, 5^{\text {th }}$, and 7 th day after EOMA cell injection for a total dose of $7.5 \mathrm{~Gy}$. On the 10th day after cell injections, mice were sacrificed and tumor volume was determined by using calipers to measure length $\times$ width $\times$ height of each tumor as previously described $(11,21)$.

\section{Immunocytochemistry and confocal microscopy}

For immunocytochemistry, cells $(50,000$ cells/well) were seeded on a coverslip. Cells were exposed to treatment after adhering to the coverslips. Post-treatment, all experimental groups were incubated in DMEM $(4.5 \mathrm{~g} / \mathrm{L}$ glucose $)$ containing $250 \mathrm{nM}$ MitoTracker ${ }^{\mathrm{TM}}$ Red CMXRos at $37^{\circ} \mathrm{C}$ for $30 \mathrm{~min}$. After staining with MitoTracker Red CMXRos, the coverslips were washed once in phosphate-buffered saline (PBS) and fixed with $4 \%$ paraformaldehyde solution made in PBS before blocking with $10 \%$ normal goat serum. Cells were then incubated with Anti-Drp1 (1:50 dilution, Cat. No. ab184247) at $4^{\circ} \mathrm{C}$ overnight. Signal was visualized by subsequent incubation with fluorescence-tagged appropriate secondary antibodies. Fluorescent-stained slides were counterstained with DAPI. Confocal microscopy was performed using a ZEISS LSM 880. Colocalization was quantified using colocalization plug-in of Zeiss software (Zen, blue edition) as per our previous reports (45-47). Pearson correlation coefficient was determined for each cell as a measure of colocalization and was expressed as mean \pm standard deviation (SD).

\section{mtDNA copy number determination}

Extracted DNAs (DNeasy Blood \& Tissue Kit; QIAGEN, Germantown, MD) from EOMA and MAE cells exposed to different conditions were used to measure mitochondrial to nuclear DNA ratio using polymerase chain reaction format as per the manufacturer's instructions (NovaQUANT ${ }^{\mathrm{TM}}$ Mouse Mitochondrial to Nuclear Ratio kit; EMD Millipore, Billerica, MA). Data were presented as mtDNA copy numbers as described previously (2).

\section{Mitochondrial mass determination}

It was done using MitoTracker ${ }^{\circledR}$ green FM. After treating with NBE or vehicle for $24 \mathrm{~h}$, cell pellet was obtained by centrifugation. Cells were resuspended in prewarmed DMEM containing $25 \mathrm{n} M$ of MitoTracker green FM (M7514; Invitrogen) and incubated for $30 \mathrm{~min}$ at $37^{\circ} \mathrm{C}$. After incubation, cells were washed two times with PBS, resuspended again in fresh medium, and analyzed by flow cytometry to access the mitochondrial mass.

\section{Statistical methods}

Two-sided two-sample $t$-tests were used to compare the differences between two groups with proper data transformation. Residual data from every test were investigated to ensure that the normality assumptions of the model were satisfied. Sensitivity analyses were also conducted using nonparametric procedures to ensure that the conclusions were robust to the selection of the statistical methods. The 
statistician was completely blinded about the study. A KaplanMeier survival analysis with log-rank test was used to compare different groups. A sample size of 5 for each group will provide at least $80 \%$ power of detecting a twofold change of each microRNA (miRNA) at type I error $\alpha=0.01$ and assuming a $25 \%$ coefficient of variation based on a two-sided, two-sample $t$-test for cell or in vitro experiment. Replication of 5 in each treatment group provided $80 \%$ power to identify the best dose that is $\sim 1.0 \mathrm{SD}$ better than the next best dose at $\alpha=0.05$. A one-sided log-rank test with a sample size of 5 per group provided $80 \%$ power at a significance level of 0.05 to detect the proportion of survival of $20 \%$ for control and $80 \%$ for combined treatment at day 14. A $p$ value of $<0.05$ was considered statistically significant for each experiment.

\section{Authors' Contributions}

Conceptualization: A.B., G.M.G., K.S., and C.K.S.; methodology: A.B., G.M.G., K.S., S.K., P.R.G., and C.K.S.; investigation and validation: A.B., A.S., P.R.G., C.M., and K.S.; formal analysis: A.B., X.P., and K.S.; writing, C.K.S. (final); original draft: A.B., G.M.G.; K.S., E.C., S.K., and C.K.S.; funding acquisition: G.M.G. and C.K.S.; resources: G.M.G. and C.K.S.; supervision: G.M.G., S.K., and C.K.S.

\section{Acknowledgments}

We thank the Ohio State University Laboratory Animal Resources for care of mice in accordance with the National Institutes of Health (NIH) guidelines. We thank Joseph Dynlacht and Joy Garrett, Radiation Oncology, IUSM, for their help in in vitro radiation experiments.

\section{Author Disclosure Statement}

The standardized NBE has been commercialized via a university start-up company commercialized as PediaBerry $^{\mathrm{TM}}$ in which C.K.S. is a shareholder. All other authors have no competing financial interests.

\section{Funding Information}

This study was supported by GM095657 to G.M.G. and, in part, by the National Institutes of Health RO1 grants GM069589, GM077185, GM108014, and NR013898 to C.K.S.

\section{Supplementary Material}

Supplementary Figure S1

Supplementary Figure S2

Supplementary Figure S3

Supplementary Figure S4

\section{References}

1. Alhazzazi TY, Kamarajan P, Xu Y, Ai T, Chen L, Verdin E, and Kapila YL. A novel sirtuin-3 inhibitor, LC-0296, inhibits cell survival and proliferation, and promotes apoptosis of head and neck cancer cells. Anticancer Res 36: 49-60, 2016.

2. Aminzadeh MA, Rogers RG, Fournier M, Tobin RE, Guan X, Childers MK, Andres AM, Taylor DJ, Ibrahim A, Ding X, Torrente A, Goldhaber JM, Lewis M, Gottlieb RA, Victor RA, and Marban E. Exosome-mediated benefits of cell therapy in mouse and human models of Duchenne muscular dystrophy. Stem Cell Reports 10: 942-955, 2018.
3. Ansari A, Rahman MS, Saha SK, Saikot FK, Deep A, and Kim KH. Function of the SIRT3 mitochondrial deacetylase in cellular physiology, cancer, and neurodegenerative disease. Aging Cell 16: 4-16, 2017.

4. Atalay M, Gordillo G, Roy S, Rovin B, Bagchi D, Bagchi $M$, and Sen CK. Anti-angiogenic property of edible berry in a model of hemangioma. FEBS Lett 544: 252-257, 2003.

5. Aune D, Giovannucci E, Boffetta P, Fadnes LT, Keum N, Norat T, Greenwood DC, Riboli E, Vatten LJ, and Tonstad S. Fruit and vegetable intake and the risk of cardiovascular disease, total cancer and all-cause mortality-a systematic review and dose-response meta-analysis of prospective studies. Int J Epidemiol 46: 1029-1056, 2017.

6. Bagul PK, Katare PB, Bugga P, Dinda AK, and Banerjee SK. SIRT-3 modulation by resveratrol improves mitochondrial oxidative phosphorylation in diabetic heart through deacetylation of TFAM. Cells 7: 235, 2018.

7. Bajzikova M, Kovarova J, Coelho AR, Boukalova S, Oh S, Rohlenova K, Svec D, Hubackova S, Endaya B, Judasova K, Bezawork-Geleta A, Kluckova K, Chatre L, Zobalova R, Novakova A, Vanova K, Ezrova Z, Maghzal GJ, Magalhaes Novais S, Olsinova M, Krobova L, An YJ, Davidova E, Nahacka Z, Sobol M, Cunha-Oliveira T, Sandoval-Acuña C, Strnad H, Zhang T, Huynh T, Serafim TL, Hozak P, Sardao VA, Koopman WJH, Ricchetti M, Oliveira PJ, Kolar F, Kubista M, Truksa J, Dvorakova-Hortova K, Pacak K, Gurlich R, Stocker R, Zhou Y, Berridge MV, Park S, Dong L, Rohlena J, and Neuzil J. Reactivation of dihydroorotate dehydrogenase-driven pyrimidine biosynthesis restores tumor growth of respiration-deficient cancer cells. Cell Metab 29: 399.e10-416.e10, 2019.

8. Banerjee J, Das Ghatak P, Roy S, Khanna S, Sequin EK, Bellman K, Dickinson BC, Suri P, Subramaniam VV, Chang CJ, and Sen CK. Improvement of human keratinocyte migration by a redox active bioelectric dressing. PLoS One 9: e89239, 2014.

9. Baskar R and Itahana K. Radiation therapy and cancer control in developing countries: can we save more lives? Int J Med Sci 14: 13-17, 2017.

10. Berkey FJ. Managing the adverse effects of radiation therapy. Am Fam Physician 82: 381-388, 394, 2010.

11. Biswas A, Clark EC, Sen CK, and Gordillo GM. Phytochemical inhibition of multidrug resistance protein-1 as a therapeutic strategy for hemangioendothelioma. Antioxid Redox Signal 26: 1009-1019, 2017.

12. Biswas A, Khanna S, Roy S, Pan X, Sen CK, and Gordillo GM. Endothelial cell tumor growth is Ape/ref-1 dependent. Am J Physiol Cell Physiol 309: C296-C307, 2015.

13. Cavalli LR, Varella-Garcia M, and Liang BC. Diminished tumorigenic phenotype after depletion of mitochondrial DNA. Cell Growth Differ 8: 1189-1198, 1997.

14. Chen Y, Fu LL, Wen X, Wang XY, Liu J, Cheng Y, and Huang J. Sirtuin-3 (SIRT3), a therapeutic target with oncogenic and tumor-suppressive function in cancer. Cell Death Dis 5: e1047, 2014.

15. Cummings BS and Schnellmann RG. Measurement of cell death in mammalian cells. Curr Protoc Pharmacol Chapter 12: Unit 12.8, 2004.

16. Deng B, Ghatak S, Sarkar S, Singh K, Das Ghatak P, Mathew-Steiner SS, Roy S, Khanna S, Wozniak DJ, McComb DW, and Sen CK. Novel bacterial diversity and fragmented eDNA identified in hyperbiofilm-forming Pseudomonas aeruginosa rugose small colony variant. iScience 23: 100827, 2020. 
17. Finley LW, Carracedo A, Lee J, Souza A, Egia A, Zhang J, Teruya-Feldstein J, Moreira PI, Cardoso SM, Clish CB, Pandolfi PP, and Haigis MC. SIRT3 opposes reprogramming of cancer cell metabolism through HIF1alpha destabilization. Cancer Cell 19: 416-428, 2011.

18. Gallego-Perez D, Pal D, Ghatak S, Malkoc V, Higuita-Castro N, Gnyawali S, Chang L, Liao WC, Shi J, Sinha M, Singh K, Steen E, Sunyecz A, Stewart R, Moore J, Ziebro T, Northcutt RG, Homsy M, Bertani P, Lu W, Roy S, Khanna S, Rink C, Sundaresan VB, Otero JJ, Lee LJ, and Sen CK. Topical tissue nano-transfection mediates non-viral stroma reprogramming and rescue. Nat Nanotechnol 12: 974-979, 2017.

19. Ganapathy-Kanniappan S and Geschwind JF. Tumor glycolysis as a target for cancer therapy: progress and prospects. Mol Cancer 12: 152, 2013.

20. Gomez de Cedron M, Vargas T, Madrona A, Jimenez A, Perez-Perez MJ, Quintela JC, Reglero G, San-Felix A, and Ramirez de Molina A. Novel polyphenols that inhibit colon cancer cell growth affecting cancer cell metabolism. J Pharmacol Exp Ther 366: 377-389, 2018.

21. Gordillo G, Fang H, Khanna S, Harper J, Phillips G, and Sen CK. Oral administration of blueberry inhibits angiogenic tumor growth and enhances survival of mice with endothelial cell neoplasm. Antioxid Redox Signal 11: 47-58, 2009.

22. Gordillo GM, Atalay M, Roy S, and Sen CK. Hemangioma model for in vivo angiogenesis: inducible oxidative stress and MCP-1 expression in EOMA cells. Methods Enzymol 352: 422-432, 2002.

23. Gordillo GM, Biswas A, Khanna S, Pan X, Sinha M, Roy S, and Sen CK. Dicer knockdown inhibits endothelial cell tumor growth via microRNA 21a-3p targeting of Nox-4. $J$ Biol Chem 289: 9027-9038, 2014.

24. Gordillo GM, Biswas A, Khanna S, Spieldenner JM, Pan X, and Sen CK. Multidrug resistance-associated protein-1 (MRP-1)-dependent glutathione disulfide (GSSG) efflux as a critical survival factor for oxidant-enriched tumorigenic endothelial cells. J Biol Chem 291: 10089-10103, 2016.

25. Hayashi J, Takemitsu M, and Nonaka I. Recovery of the missing tumorigenicity in mitochondrial DNA-less HeLa cells by introduction of mitochondrial DNA from normal human cells. Somat Cell Mol Genet 18: 123-129, 1992.

26. Heger V, Tyni J, Hunyadi A, Horakova L, LahtelaKakkonen M, and Rahnasto-Rilla M. Quercetin based derivatives as sirtuin inhibitors. Biomed Pharmacother 111: 1326-1333, 2019.

27. Hill BG, Benavides GA, Lancaster JR, Jr., Ballinger S, Dell'Italia L, Jianhua Z, and Darley-Usmar VM. Integration of cellular bioenergetics with mitochondrial quality control and autophagy. Biol Chem 393: 1485-1512, 2012.

28. Jaillet C, Morelle W, Slomianny MC, Paget V, Tarlet G, Buard V, Selbonne S, Caffin F, Rannou E, Martinez P, Francois A, Foulquier F, Allain F, Milliat F, and Guipaud O. Radiation-induced changes in the glycome of endothelial cells with functional consequences. Sci Rep 7: 5290, 2017.

29. Jegasothy SM, Zabolotniaia V, and Bielfeldt S. Efficacy of a new topical nano-hyaluronic acid in humans. J Clin Aesthet Dermatol 7: 27-29, 2014.

30. Kim HS, Patel K, Muldoon-Jacobs K, Bisht KS, AykinBurns N, Pennington JD, van der Meer R, Nguyen P, Savage J, Owens KM, Vassilopoulos A, Ozden O, Park SH, Singh KK, Abdulkadir SA, Spitz DR, Deng CX, and Gius D. SIRT3 is a mitochondria-localized tumor suppressor required for maintenance of mitochondrial integrity and metabolism during stress. Cancer Cell 17: 41-52, 2010.
31. Kim J, Yang G, Kim Y, Kim J, and Ha J. AMPK activators: mechanisms of action and physiological activities. Exp Mol Med 48: e224, 2016.

32. Klimova N, Long A, and Kristian T. Nicotinamide mononucleotide alters mitochondrial dynamics by SIRT3-dependent mechanism in male mice. J Neurosci Res 97: 975-990, 2019.

33. Kotecha R, Takami A, and Espinoza JL. Dietary phytochemicals and cancer chemoprevention: a review of the clinical evidence. Oncotarget 7: 52517-52529, 2016.

34. Li S, Dou X, Ning H, Song Q, Wei W, Zhang X, Shen C, Li J, Sun C, and Song Z. Sirtuin 3 acts as a negative regulator of autophagy dictating hepatocyte susceptibility to lipotoxicity. Hepatology 66: 936-952, 2017.

35. Maycotte P, Marín-Hernández A, Goyri-Aguirre M, AnayaRuiz M, Reyes-Leyva J, and Cortés-Hernández P. Mitochondrial dynamics and cancer. Tumour Biol 39: $1010428317698391,2017$.

36. Mookerjee SA, Gerencser AA, Nicholls DG, and Brand MD. Quantifying intracellular rates of glycolytic and oxidative ATP production and consumption using extracellular flux measurements. J Biol Chem 292: 7189-7207, 2017.

37. Morais R, Zinkewich-Peotti K, Parent M, Wang H, Babai $\mathrm{F}$, and Zollinger $\mathrm{M}$. Tumor-forming ability in athymic nude mice of human cell lines devoid of mitochondrial DNA. Cancer Res 54: 3889-3896, 1994.

38. Nayler SJ, Rubin BP, Calonje E, Chan JK, and Fletcher CD. Composite hemangioendothelioma: a complex, lowgrade vascular lesion mimicking angiosarcoma. Am J Surg Pathol 24: 352-361, 2000.

39. Parrado-Fernandez C, Sandebring-Matton A, RodriguezRodriguez P, Aarsland D, and Cedazo-Minguez A. Anthocyanins protect from complex I inhibition and APPswe mutation through modulation of the mitochondrial fission/fusion pathways. Biochim Biophys Acta 1862: 2110-2118, 2016.

40. Prencipe FP, Bruni R, Guerrini A, Rossi D, Benvenuti S, and Pellati F. Metabolite profiling of polyphenols in Vaccinium berries and determination of their chemopreventive properties. J Pharm Biomed Anal 89: 257-267, 2014.

41. Riccardi C and Nicoletti I. Analysis of apoptosis by propidium iodide staining and flow cytometry. Nat Protoc 1: 1458-1461, 2006.

42. Rink C, Gnyawali S, Peterson L, and Khanna S. Oxygeninducible glutamate oxaloacetate transaminase as protective switch transforming neurotoxic glutamate to metabolic fuel during acute ischemic stroke. Antioxid Redox Signal 14: 1777-1785, 2011.

43. Samant SA, Zhang HJ, Hong Z, Pillai VB, Sundaresan NR, Wolfgeher D, Archer SL, Chan DC, and Gupta MP. SIRT3 deacetylates and activates OPA1 to regulate mitochondrial dynamics during stress. Mol Cell Biol 34: 807-819, 2014.

44. Shimura T, Sasatani M, Kawai H, Kamiya K, Kobayashi J, Komatsu K, and Kunugita N. A comparison of radiationinduced mitochondrial damage between neural progenitor stem cells and differentiated cells. Cell Cycle 16: 565-573, 2017.

45. Singh K, Pal D, Sinha M, Ghatak S, Gnyawali SC, Khanna S, Roy $S$, and Sen CK. Epigenetic modification of microRNA-200b contributes to diabetic vasculopathy. Mol Ther 25: 2689-2704, 2017.

46. Singh K, Sinha M, Pal D, Tabasum S, Gnyawali SC, Khona D, Sarkar S, Mohanty SK, Soto-Gonzalez F, Khanna S, Roy S, and Sen CK. Cutaneous epithelial to mesenchymal transition activator ZEB1 regulates wound angiogenesis and closure in a glycemic status dependent manner. Diabetes 68: 2175-2190, 2019. 
47. Sinha M, Sen CK, Singh K, Das A, Ghatak S, Rhea B, Blackstone B, Powell HM, Khanna S, and Roy S. Direct conversion of injury-site myeloid cells to fibroblast-like cells of granulation tissue. Nat Commun 9: 936, 2018.

48. Stevens JF, Revel JS, and Maier CS. Mitochondria-centric review of polyphenol bioactivity in cancer models. Antioxid Redox Signal 29: 1589-1611, 2018.

49. Suárez-Rivero JM, Villanueva-Paz M, de la Cruz-Ojeda P, de la Mata M, Cotán D, Oropesa-Ávila M, de Lavera I, Álvarez-Córdoba M, Luzón-Hidalgo R, and SánchezAlcázar JA. Mitochondrial dynamics in mitochondrial diseases. Diseases 5: 1, 2016.

50. Sullivan LB, Gui DY, Hosios AM, Bush LN, Freinkman E, and Vander Heiden MG. Supporting aspartate biosynthesis is an essential function of respiration in proliferating cells. Cell 162: 552-563, 2015.

51. Sullivan LB, Gui DY, and Vander Heiden MG. Altered metabolite levels in cancer: implications for tumour biology and cancer therapy. Nat Rev Cancer 16: 680-693, 2016.

52. Thomasset SC, Berry DP, Garcea G, Marczylo T, Steward WP, and Gescher AJ. Dietary polyphenolic phytochemicalspromising cancer chemopreventive agents in humans? A review of their clinical properties. Int J Cancer 120: 451-458, 2007.

53. Viale A, Corti D, and Draetta GF. Tumors and mitochondrial respiration: a neglected connection. Cancer Res 75: 3685-3686, 2015.

54. Vieira Dias J, Gloaguen C, Kereselidze D, Manens L, Tack $\mathrm{K}$, and Ebrahimian TG. Gamma low-dose-rate ionizing radiation stimulates adaptive functional and molecular response in human aortic endothelial cells in a threshold-, dose-, and dose rate-dependent manner. Dose Response 16: $1559325818755238,2018$.

55. Wallace DC. Mitochondria and cancer. Nat Rev Cancer 12: 685-698, 2012.

56. Wang LS and Stoner GD. Anthocyanins and their role in cancer prevention. Cancer Lett 269: 281-290, 2008.

57. Wang M, Smith K, Yu Q, Miller C, Singh K, and Sen CK. Mitochondrial connexin 43 in sex-dependent myocardial responses and estrogen-mediated cardiac protection following acute ischemia/reperfusion injury. Basic Res Cardiol 115: 1, 2019.

58. Wang X, Ouyang Y, Liu J, Zhu M, Zhao G, Bao W, and Hu FB. Fruit and vegetable consumption and mortality from all causes, cardiovascular disease, and cancer: systematic review and dose-response meta-analysis of prospective cohort studies. BMJ 349: g4490, 2014.

59. Weiss SW and Enzinger FM. Epithelioid hemangioendothelioma: a vascular tumor often mistaken for a carcinoma. Cancer 50: 970-981, 1982.

60. Wen YA, Xiong X, Scott T, Li AT, Wang C, Weiss HL, Tan L, Bradford E, Fan TWM, Chandel NS, Barrett TA, and Gao T. The mitochondrial retrograde signaling regulates Wnt signaling to promote tumorigenesis in colon cancer. Cell Death Differ 26: 1955-1969, 2019.

61. Yang B, Fu X, Shao L, Ding Y, and Zeng D. Aberrant expression of SIRT3 is conversely correlated with the progression and prognosis of human gastric cancer. Biochem Biophys Res Commun 443: 156-160, 2014.

62. Zhang J, Meruvu S, Bedi YS, Chau J, Arguelles A, Rucker $\mathrm{R}$, and Choudhury M. Pyrroloquinoline quinone increases the expression and activity of Sirt 1 and -3 genes in HepG2 cells. Nutr Res 35: 844-849, 2015.

63. Zhang J, Wu B, Zhou GQ, Zhang RS, Wei X, Yu B, Lu ZF, Ma $\mathrm{HH}$, Shi QL, and Zhou XJ. Composite hemangioendothelioma arising from the kidney: case report with review of the literature. Int J Clin Exp Pathol 6: 1935-1941, 2013.

64. Zhong J, Rajaram N, Brizel DM, Frees AE, Ramanujam N, Batinic-Haberle I, and Dewhirst MW. Radiation induces aerobic glycolysis through reactive oxygen species. Radiother Oncol 106: 390-396, 2013.

65. Zorova LD, Popkov VA, Plotnikov EY, Silachev DN, Pevzner IB, Jankauskas SS, Babenko VA, Zorov SD, Balakireva AV, Juhaszova M, Sollott SJ, and Zorov DB. Mitochondrial membrane potential. Anal Biochem 552: 50 59, 2018.

Address correspondence to: Prof. Gayle M. Gordillo Department of Surgery

Indiana Center for Regenerative Medicine and Engineering Indiana University School of Medicine Indianapolis, IN 46202

USA

E-mail: gmgordil@iu.edu

Date of first submission to ARS Central, February 7, 2020; date of final revised submission, June 11, 2020; date of acceptance, June 25, 2020.

$\begin{aligned} & \quad \text { Abbreviations Used } \\ & \Delta \Psi \mathrm{m}=\text { mitochondrial membrane potential } \\ & 2 \mathrm{D}=\text { two dimensional } \\ & \mathrm{AMPK}=\text { AMP-activated protein kinase } \\ & \mathrm{ATP}=\text { adenosine triphosphate } \\ & \mathrm{BCA}=\text { bicinchoninic acid } \\ & \mathrm{DMEM}=\text { Dulbecco's modified Eagle's medium } \\ & \mathrm{DMSO}=\text { dimethyl sulfoxide } \\ & \mathrm{Drp} 1=\text { dynamin-related protein } 1 \\ & \mathrm{ECAR}=\text { extracellular acidification rate } \\ & \mathrm{ELISA}=\text { enzyme-linked immunosorbent assay } \\ & \mathrm{EOMA}=\text { mouse hemangioendothelioma endothelial } \\ & \mathrm{HE}=\text { hemangioendothelioma } \\ & \mathrm{LDH}=\text { lactate dehydrogenase } \\ & \mathrm{MAE}=\text { mouse aortic endothelial } \\ & \mathrm{MFF}=\text { mitochondrial fission factor } \\ & \mathrm{Mfn}-2=\text { mitofusin } 2 \\ & \mathrm{mtDNA}=\text { mitochondrial DNA } \\ & \mathrm{NBE}=\text { natural berry extract } \\ & \mathrm{OCR}=\text { oxygen consumption rate } \\ & \mathrm{OXPHOS}=\text { oxidative phosphorylation } \\ & \mathrm{PBS}=\text { phosphate-buffered saline } \\ & \mathrm{PQQ}=\text { pyrroloquinoline quinone } \\ & \mathrm{ROS}=\text { reactive oxygen species } \\ & \mathrm{SD}=\text { standard deviation } \\ & \mathrm{SIRT} 3=\text { sirtuin } 3 \\ & \mathrm{XRT}=\text { X-ray therapy } \\ &\end{aligned}$

